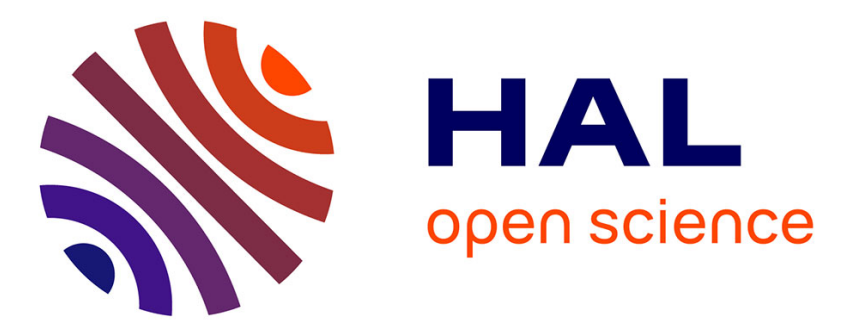

\title{
Producing Genomic Sequences after Genome Scaffolding with Ambiguous Paths: Complexity, Approximation and Lower Bounds
}

Tom Davot, Annie Chateau, Rodolphe Giroudeau, Mathias Weller, Dorine Tabary

\section{To cite this version:}

Tom Davot, Annie Chateau, Rodolphe Giroudeau, Mathias Weller, Dorine Tabary. Producing Genomic Sequences after Genome Scaffolding with Ambiguous Paths: Complexity, Approximation and Lower Bounds. Algorithmica, 2021, 10.1007/s00453-021-00819-6 . lirmm-03218029v1

\section{HAL Id: lirmm-03218029 \\ https://hal-lirmm.ccsd.cnrs.fr/lirmm-03218029v1}

Submitted on 5 May 2021 (v1), last revised 3 Jun 2021 (v2)

HAL is a multi-disciplinary open access archive for the deposit and dissemination of scientific research documents, whether they are published or not. The documents may come from teaching and research institutions in France or abroad, or from public or private research centers.
L'archive ouverte pluridisciplinaire HAL, est destinée au dépôt et à la diffusion de documents scientifiques de niveau recherche, publiés ou non, émanant des établissements d'enseignement et de recherche français ou étrangers, des laboratoires publics ou privés. 


\title{
Producing genomic sequences after genome scaffolding with ambiguous paths
}

\author{
Tom Davot ${ }^{1}$, Annie Chateau ${ }^{1}$, Rodolphe Giroudeau ${ }^{1}$, Mathias $_{\text {Weller }}^{2}$, Dorine \\ Tabary $^{3}$ \\ 1 LIRMM - CNRS UMR 5506 - Montpellier, France \\ 2 CNRS, LIGM (UMR 8049), Champs-s/-Marne, France \\ 3 MIPS EA 2332- Mulhouse, France \\ \{davot, chateau, rgirou\}@lirmm.fr, mathias.weller@u-pem.fr, \\ dorine.tabary@uha.fr
}

\begin{abstract}
Scaffolding is the final step in assembling Next Generation Sequencing data, in which pre-assembled contiguous regions ("contigs") are oriented and ordered using information that links them (for example, mapping of paired-end reads). As the genome of some species is highly repetitive, we allow placing some contigs multiple times, thereby generalizing established computational models for this problem. We study the subsequent problems induced by the translation of solutions of the model back to actual sequences, proposing models and analyzing the complexity of the resulting computational problems. We find both polynomialtime and $\mathcal{N} \mathcal{P}$-hard special cases like planarity or bounded degree. Finally, we propose two polynomial-time approximation algorithms according to cut/weight score.
\end{abstract}

\section{Introduction}

Context and motivation. Genomic data are of major importance in numerous aspects of research and applications in biology and computational biology. Their production is massively encouraged by industrial and academic actors, who use them in various ways [?]. These data are produced by so-called sequencers, who output (typically millions of) reads, that is, tiny subsequences of DNA that need to be "assembled" in order to get the target genome. The genome, once stabilized, is stored in huge databases and is made available to the community for further analysis. A high-quality genome is of paramount importance to the accuracy of further methods (such as genome comparison, gene inference, studies on the order of given markers, etc). Thus, it is crucial to provide genomes as complete and error-free as possible.

Preprint version.

The final authenticated version is available online at https://doi.org/10.1007/ s00453-021-00819-6. 
Assembly and scaffolding steps. The operation consisting in merging reads together to produce longer sequences is called genome assembly. Many methods and tools propose to assemble Next Generation Sequencing (NGS) reads into genomes, metagenomes or transcriptomes, most of them modeling sequences through graphs (assembly graphs, k-mer graphs, A-Bruijn graphs, etc.) [? ? ? ? ? ? ? ]. Those tools are compared and evaluated through benchmarks of different origins [? ?]. A recent state-of-the art about genome assembly has been compiled by Phillippy [? ]. However, assembly software typically has trouble dealing with repetitive (parts of the) genomes [? ? ?] and, therefore, output a collection of "contiguous regions" (contigs), that is, large chunks of DNA covering most of the genome. Unfortunately, nearly all "known" genomes are in a thusly fragmented state; some mammalian genomes reach hundreds of contigs per chromosome [? ]. To overcome this issue, an additional step, called scaffolding, intends to reduce the fragmentation using additional data (paired-end reads, long reads, phylogenetic information, etc.). To this end, scaffolding software computes the most likely order and relative orientation of these contigs along the genome and, if possible, fills gaps between them [? ? ? ?]. Scaffolding methods are also mainly based on various models of graphs, representing the way additional data link contigs. However, as with reads, the target genome may contain multiple (inverted) copies of an entire contig, like the well-known and described large inverted repeat region in chloroplast genomes [?], and many scaffolders are incapable of handling these repeats. Recent techniques use third-generation sequencing data [?] to resolve these repeats, but it requires resequencing the large amount of available, highly fragmented genomes. A possible way to solve the problem without resequencing is to deduce multiplicities of contigs using external information (such as read-coverage) and take this multiplicity into account when scaffolding.

Linearization of the solution. Scaffolding leads to a set of paths and cycles in a scaffolding graph. When a repeated contig is involved in several paths corresponding to distinct parts of the genome, it is impossible to distinguish between the copies, and paths collapse into non-linear structures (see Figures 1 and 2, requiring some definitions of Section 2). This solution structure is informative per se and could be used as it comes, but it presents sequences non-linearly. However, the standard representation of scaffolds are linear sequences of nucleotides. Thus, we need to linearize the solution graph, that is, resolve the ambiguities arising from the indistinguishability among the copies of each repeated contig, This is the main subject of this work. It turns out that the most straight-forward linearization strategies may produce chimeric sequences, and we show that the ones avoiding chimeras in a parsimonious way are $\mathcal{N} \mathcal{P}$-hard to compute (for reasonable scoring). In particular, our model is an edge-deletion problem (called Semi-Brutal CUt) concentrated on extremities of ambiguities in a "solution graph" whose structure influences the computational tractability of the problem (see Table 1 and Table 2 for a summary).

Organization of this article. The next section is devoted to definitions and the descriptions of problems related to scaffolding and linearization. In Section 3, the 
Table 1: Overview of complexity results for Semi-Brutal Cut.

\begin{tabular}{|c|c|c|c|}
\hline Topologies & Type of cut & Complexi & \\
\hline Complete bipartite & \multirow{3}{*}{ Cut score } & \multirow{5}{*}{ Linear } & Theorem 4 \\
\hline Complete & & & Theorem 5 \\
\hline Cobipartite & & & Theorem 6 \\
\hline Trees & \multirow{3}{*}{ All } & & \begin{tabular}{|l} 
Theorem 2 \\
\end{tabular} \\
\hline$\Delta \leq 2$ & & & Theorem 3 \\
\hline $\mathcal{G}^{\prime 1}$ & & \multirow{3}{*}{$\mathcal{N P}$-hard } & Theorem 7 \\
\hline Supergraphs of $\mathcal{G}^{\prime}$ & Weight score & & \begin{tabular}{|l|} 
Corollary 2 \\
\end{tabular} \\
\hline \multirow{2}{*}{$\begin{array}{l}\text { Split } \\
\text { graphs }\end{array}$} & \multirow[b]{2}{*}{ Cut score } & & Theorem 8 \\
\hline & & $\begin{array}{l}\text { No } 2^{o(n)} \text { algorithm } \\
\quad(\text { under } \mathcal{E} \mathcal{T} \mathcal{H} \text { ) }\end{array}$ & Corollary 3 \\
\hline
\end{tabular}

Table 2: Overview of lower and upper bounds for Semi-Brutal Cut.

\begin{tabular}{|c|c|c|c|c|c|}
\hline Topologies & Type of Cut & Hypothesis & \multicolumn{2}{|c|}{ Lower bound } & Upper bound \\
\hline $\mathcal{G}^{\prime 1}$ & \multirow{7}{*}{ Cut score } & \multirow{6}{*}{$\mathcal{P} \neq \mathcal{N} \mathcal{P}$} & $1.00009 \ldots$ & Theorem 9 & \multirow{7}{*}{$\begin{array}{c}\text { 4-approx } \\
\text { Theorem } 15\end{array}$} \\
\hline Subcubic & & & $1.00041 \ldots$ & Theorem 12 & \\
\hline$\Delta \leq 4$ & & & $1.0069 \ldots$ & \multirow{3}{*}{ Theorem 14} & \\
\hline$\Delta \leq 5$ & & & $1.0128 \ldots$ & & \\
\hline$\Delta \leq 6$ & & & $1.0138 \ldots$ & & \\
\hline \multirow{2}{*}{$\begin{array}{c}\text { General, } \\
\text { Split graphs }\end{array}$} & & & $1.360 \ldots$ & \multirow{2}{*}{ Theorem 13} & \\
\hline & & 7 & $2-\epsilon$ & & \\
\hline \multirow{3}{*}{ General } & \multirow{3}{*}{$\begin{array}{l}\text { Weight } \\
\text { score }\end{array}$} & Uye & $1.01887 \ldots$ & \multirow{2}{*}{ Theorem 10} & \multirow{3}{*}{$\begin{array}{c}\text { 2-approx } \\
\text { Theorem } 16\end{array}$} \\
\hline & & $\mathcal{P} \neq \mathcal{N} \mathcal{P}$ & $1.01515 \ldots$ & & \\
\hline & & & $1.00016 \ldots$ & Theor & \\
\hline
\end{tabular}

1. Bipartite, planar and subcubic

notion of unambiguous solutions is explained. In Section 4, we describe several reduction rules yielding a simplified version of the linearization problem. The polynomial cases are developed in Section 5 whereas the hardness cases are presented in Section 6. The non-approximability results are given in Section 7 and, in the last section, several polynomial-time approximation algorithms are developed.

\section{Obtaining Sequences From Solution Graphs}

We consider simple, loopless graphs. Let $G$ be such a graph. We denote by $V(G)$ and $E(G)$ the set of vertices and edges of $G$, respectively (or $V$ and $E$ if no ambiguity occurs). The degree of a vertex $v$ is the number of edges incident to $v$ and is denoted by $\operatorname{deg}_{G}(v)$. The set of neighbors of $v$ is denoted by $N(v)$. The maximum degree of $G$ is $\Delta(G)$. Consider a set of contigs $\mathcal{C}=\left\{C_{1}, \ldots, C_{n}\right\}$ and a set of weighted links between contig extremities (obtained from paired-end reads mapping). This can be represented by a graph $G$ containing, for each contig $C_{i}$, vertices $u_{i}$ and $v_{i}$ representing the extremities of $C_{i}$, an edge $u_{i} v_{i}$ repre- 
senting the contig $C_{i}$ (contig edge), and weighted links between contig extremities (non-contig edges). The contig edges form a perfect matching $M^{*}$ in $G$. In the following of the paper, we refer to them as matching edges. The weight function $\omega$ is defined on non-matching edges and symbolizes, roughly, the amount of confidence that we have in the link. We call such a graph a scaffold graph. For the matching $M^{*}$ and a vertex $u$, we define $M^{*}(u)$ as the unique vertex $v$ with $u v \in M^{*}$. Slightly abusing notation, we sometimes consider graphs as sets of edges. Then, a path $p$ is alternating with respect to a matching $M^{*}$ if, for all vertices $u$ of $p$, also $M^{*}(u)$ is a vertex of $p$ (see Figure 3a for an example). Then, a linear (circular) chromosome in the target genome is reflected as an alternating path (cycle) in $G$. One might now ask for the most parsimonious way (that is, discarding as little weight as possible) of inferring a given number $\sigma_{\mathrm{p}}$ of linear (and $\sigma_{\mathrm{c}}$ of circular) chromosomes that, together, make up $\mathcal{C}$. This problem has been modeled as the following, computationally hard problem [? ?].

SCAFFolding (SCA)

Input: A scaffold graph $\left(G, M^{*}, \omega\right)$ and $\sigma_{\mathrm{p}}, \sigma_{\mathrm{c}}, k \in \mathbb{N}$.

Question: Is there some $S \subseteq E(G) \backslash M^{*}$ such that $S \cup M^{*}$ is a collection of $\leq \sigma_{\mathrm{p}}$ alternating paths and $\leq \sigma_{\mathrm{c}}$ alternating cycles and $\omega(S) \geq k$ ?

To work with multiplicities, we consider walks instead of paths. A length- $\ell$ walk in a graph $G$ is a sequence $\left(u_{0}, u_{1}, \ldots, u_{\ell}\right)$ of vertices in $V(G)$ such that, for each two consecutive vertices $u_{i}$ and $u_{i+1}$ in the sequence, we have $u_{i} u_{i+1} \in E(G)$. The walk is called closed if $u_{0}=u_{\ell}$ and it is called alternating with respect to a perfect matching $M^{*}$ in $G$ if (a) $u_{i} u_{i+1} \in M^{*}$ if and only if $i$ is even, and (b) $\ell$ is even if and only if the walk is closed. We will consider walks as multisets of edges. For any multiset $W$, let $\chi_{W}(e)$ be the number of times that $e$ occurs in $W$ and let $\omega(W):=\sum_{e \in W} \chi_{W}(e) \omega(e)$. When working with multiplicities, each matching edge $e$ of the scaffold graph has a multiplicity $m^{\prime}(e)$. For matching edges, this can be read from the data as described in the introduction. Then, the scaffolding problem with multiplicities is the following:

Scaffolding with Multiplicities (MSCA)

Input: A scaffold graph $\left(G, M^{*}, \omega, m^{\prime}\right)$ and $\sigma_{\mathrm{p}}, \sigma_{\mathrm{c}}, k \in \mathbb{N}$.

Question: Is there a multiset $S$ of $\leq \sigma_{\mathrm{c}}$ closed and $\leq \sigma_{\mathrm{p}}$ non-closed alternating walks in $\left(G, M^{*}, \omega\right)$ such that each $e \in M^{*}$ occurs exactly $m^{\prime}(e)$ times in walks of $S$ and $\omega(S) \geq k$ ?

Obtaining solutions for MSCA is not the topic of this work. Instead, we consider a solution for MSCA, that is, a multiset $S$ of alternating walks in $G$ such that each $e \in M^{*}$ occurs exactly $m^{\prime}(e)$ times in walks of $S$. From $S$, we reconstruct a solution graph $h^{4} \operatorname{sol}(S):=\left(G^{*}, M^{*}, \omega, m\right)$ by "merging" all walks of $S$, that is, $G^{*}$ contains exactly the edges $e$ of $G$ that occur in walks of $S$ and $m(e)=\sum_{W \in S} \chi_{W}(e)$ is the number of their occurrences. Note that the func-

\footnotetext{
${ }^{4}$ Solution graphs differ from scaffold graphs in that the multiplicity function is defined on all edges and not just on matching edges.
} 


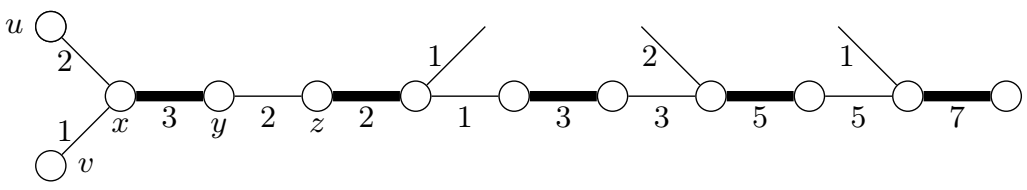

Fig. 1: Walks in a scaffold graph (not drawn) give a solution graph (drawn) with multiplicities. Matching edges are bold. The only ambiguous path is $(x, y)$. It is ambiguous because it can be decomposed into $\{(\ldots, u, x, y, z, \ldots),(\ldots, u, x, y, z, \ldots),(\ldots, v, x, y)\} \quad$ or $\{(\ldots, u, x, y),(\ldots, u, x, y, z, \ldots),(\ldots, v, x, y, z, \ldots)\}$. Removing all nonmatching edges incident with $x$ or all non-matching edges incident with $y$ destroys all ambiguous paths.

tion $m$ is defined on every edges (contrary to $m^{\prime}$ which is only defined on the matching edges) and that for any matching edge $e$, we have $m(e)=m^{\prime}(e)$. We also say that $\operatorname{sol}(S)$ is made up of $S$. This merge translates the fact that copies of repeated contigs cannot be distinguished using information from the scaffold graph. Any set of walks making up this solution graph is also a solution of Scaffolding With Multiplicities with the same optimal score. The solution graph is, in fact, a manner of representing all the optimal solutions. Any arbitrary choice between them could lead to chimeric scaffolds. ${ }^{5}$ Indeed, the problem is that sol is not necessarily injective. For example, suppose that the edge $x y$ in Figure 1 is used in three walks, two of which contain the vertex $z$. As $x$ is incident to different non-matching edges, one of the three walks differs from the other two, but it cannot be determined whether or not it is the same walk that avoids $z$. See also Figure 2 for an example with sequences and Figure 3 for an example of a scaffold graph leading to a solution graph with ambiguous sequences. This notion is captured in the following definition.

Definition 1. Let $A$ be an alternating path between $u$ and $v$ or an alternating cycle in a solution graph. If all edges of $A$ have the same multiplicity $\mu$ (that is, $m(e)=\mu$ for all $e \in A$ ), then $A$ is called $\mu$-uniform (or simply uniform if $\mu$ is unknown). Further,

1. if $A$ is an alternating $\mu$-uniform cycle and $\mu>1$, or

2. if $A$ is an alternating $\mu$-uniform $u$-v-path and each of $u$ and $v$ is incident with a non-matching edge of multiplicity strictly less than $\mu$,

then $A$ is called ambiguous.

An example of ambiguous path is depicted in Figure 1. Roughly speaking, the problem is that there are many ways of pairing up sequences on each end of ambiguous paths and that the number of cycles is undefined in ambiguous cycles. Interestingly, ambiguous paths and cycles are enough to characterize ambiguity of solution graphs (proof in Section 3).

\footnotetext{
${ }^{5}$ A sequences is called chimeric if it does not occur in the target genome, but is made up of chunks picked from different chromosomes or regions of the genome.
} 


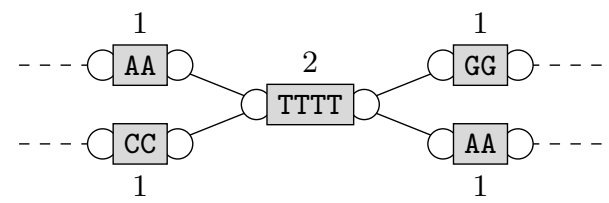

Fig. 2: A schema illustrating solution ambiguity: from the solution graph alone, we cannot tell whether the target genome contains (1) AATTTTGG and CCTTTAA or (2) AATTTTAA and CCTTTTGG. As methods "ignore" and "clever" choose one of the two, they may produce wrong sequences. Method "brutal" removes all four edges incident with the matching edges TTTT and "semi-brutal" removes either the left or the right pair of edges. Note that this problem does not go away if we require the solution to be represented as a collection of walks, as this collection will just represent one of the arbitrary choices.

Theorem 1. Let $G^{*}$ be a solution graph. Then, $G^{*}$ is made up of a unique multiset of alternating walks if and only if $G^{*}$ does not contain ambiguous paths or cycles.

For biological applications, the representation as solution graph is not satisfying. Instead, it is necessary to translate the solution into sequences. However, each solution $S$ corresponds to a different collection of sequences which, without additional external knowledge, are equally likely from a biological point of view. For a solution graph $G^{*}$, we let $\operatorname{sol}^{-1}\left(G^{*}\right)$ denotes the set of multisets $S$ of walks with $\operatorname{sol}(S)=G^{*}$. Theorem 1 states that $\left|\operatorname{sol}^{-1}(S)\right|=1$ if and only if $G^{*}$ does not contain ambiguous paths or cycles. However, if the solution graph does contain ambiguous paths, we propose the following strategies for its translation into sequences.

Ignore. Choose an arbitrary multiset of walks making up $G^{*}$. In this case, we preserve the weight of the solution, but there is no way to distinguish between the elements of $\operatorname{sol}^{-1}\left(G^{*}\right)$ and the arbitrary choice could lead to an erroneous solution, biologically speaking. Indeed, there is a risk to produce a chimeric sequence, and this strategy has to be put aside in a bioinformatic context.

Clever. Choose walks that optimize some criterion (i.e. N506 ). This strategy consists in finding, among all solutions of maximal weight in $\operatorname{sol}^{-1}\left(G^{*}\right)$, one which maximizes this global criterion. Again, this strategy induces a risk to produce chimeric sequences, and we will not consider it any further.

Brutal. Isolate ambiguous paths by removing all non-matching edges incident to their extremities. Remove one non-matching edge in each ambiguous cycle to transform it into a uniform path.

Semi-brutal. Choose a proper set of endpoints of ambiguous path and remove all non-matching edges incident to it. Remove one non-matching edge in each ambiguous cycle to transform it into a uniform path.

\footnotetext{
${ }^{6} \mathrm{~N} 50$ is a statistical measure on contig lengths: given a set of contigs, the N50 is defined as the sequence length of the shortest contig at $50 \%$ of the total genome length.
} 
We will focus on methods "brutal" and "semi-brutal" as the other methods may produce chimeric sequences (See Figure 2). However, since we remove edges, the final scaffold may not have maximum weight among all uniquely linearizable solutions, and we will discuss this point. Method "brutal" can be executed in polynomial time, but it may decrease the weight of the solution drastically. Method "semi-brutal" forces us to make a choice each time we encounter an ambiguous path, and we might want to choose "wisely", that is, destroy ambiguous paths in a way that optimizes a scoring. Let $v$ be either an extremity of an ambiguous path or a vertex of an ambiguous cycle, we sometimes say "to cut $v$ ", by which we mean removing all non-matching edges incident to it, and in that case $v$ is denoted as a cut. Thus, the following problem arises:

Semi-Brutal Cut (SBC)

Input: A solution graph $\left(G^{*}, M^{*}, \omega, m\right)$ and some $k \in \mathbb{N}$.

Question: Is there a set $X$ of cuts of $G^{*}$ which destroys all ambiguous paths and the score of $X$ is at most $k$ ?

In Section 4, we show how to simplify the problem statement. Notice that separating Semi-Brutal Cut from Scaffolding with Multiplicities is necessary in order to avoid the production of a chimeric sequence, as explained in Figure 3. Several possible scoring functions seem sensible to optimize:

Cut score. Pay one per cut: $\operatorname{score}(X):=|X|$.

Path score. Pay one for each multiplicity that is cut: $\operatorname{score}(X):=\sum\left\{m(u v) \mid u v \in E\left(G^{*}\right) \backslash M^{*} \wedge\{u, v\} \cap X \neq \varnothing\right\}$.

Weight score. Pay the total weight of edges that are cut: $\operatorname{score}(X):=\sum\left\{m(u v) \cdot \omega(u v) \mid u v \in E\left(G^{*}\right) \backslash M^{*} \wedge\{u, v\} \cap X \neq \varnothing\right\}$.

Note that, from the perspective of computational complexity, the path score is a special case of the weight score, since we can just set $\omega(e)=1$ for all edges $e$. Thus, when saying "both scores" we refer to cut and weight score. Unfortunately, it turns out that all these variants are $\mathcal{N} \mathcal{P}$-hard (see Section 6).

\section{Unambiguous Solutions}

We show in this section that the solution graph $G^{*}$ has to be free of ambiguous paths and cycles in order to be uniquely deconstructable into walks that make up $G^{*}$. To this end, we present a reduction rule whose application does not change unique deconstructability (indeed, it does not change $\left|\mathrm{sol}^{-1}\left(G^{*}\right)\right|$ ). 


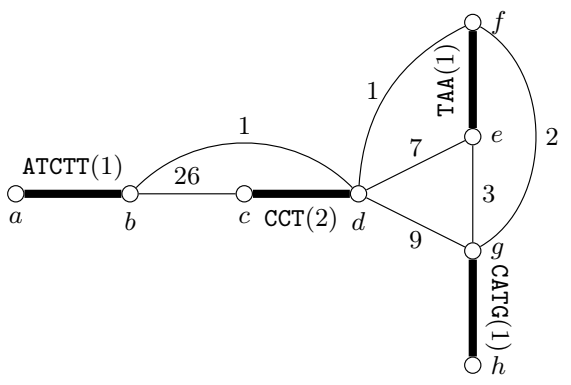

(a) Scaffold graph. This graph illustrates relationship between four contigs, figured by bold edges $a b, c d, e f$ and $g h$. Labels on these edges show the sequence of the contigs, and their mutliplicity (in parenthesis). Edge $c d$, whose sequence is CCT, has multiplicity two. Other contigs have multiplicity one. Links between contigs are labeled by their weight.

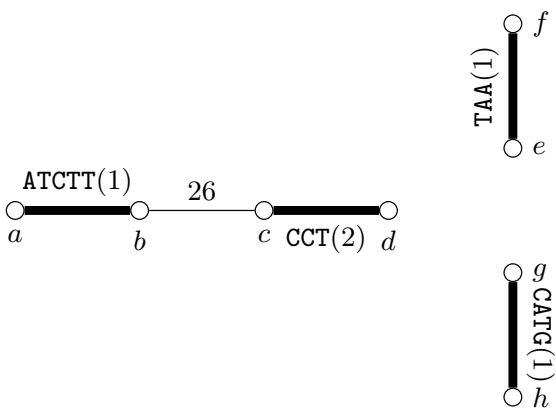

(c) Linearization using SEmI-Brutal Cut. Brutal cut would provide a set of four independent sequences of total weight zero (the initial set of contigs), whereas SEmi-Brutal Cut with weightscore provides a unique set of four sequences $\{$ ATCCT..CCT, CCT, TAA, CATG $\}$, and weight 26 (minimal weight-score 16). After solving successively MSCA (with $\sigma_{p}=2$ and $\sigma_{c}=0$ ) and SBC, the solution is compatible with the initial hypothesis. The only ambiguous path is the matching edge $c d$ and the cut vertex is $d$.

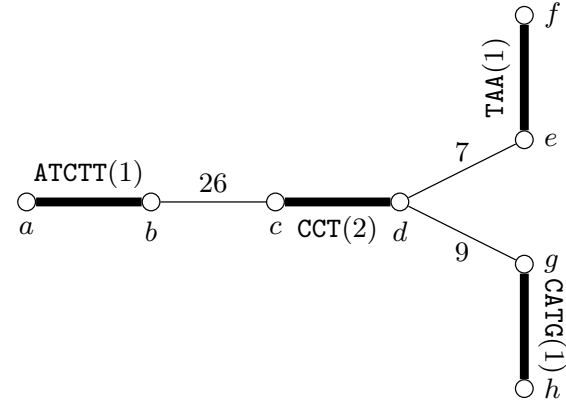

(b) Solution graph after solving SCAFFolding With Multiplicities. The solution graph is obtained as a solution for the MSCA instance asking for two open walks with total weight $\geq 42$. In the solution graph, the contig of multiplicity two labeled CCT constitutes an ambiguous path, yielding two possible sets of sequences $\{$ ATCCT..CCT..TAA, CCT..CATG $\}$ and \{ATCCT..CCT..CATG, CCT..TAA $\}$.

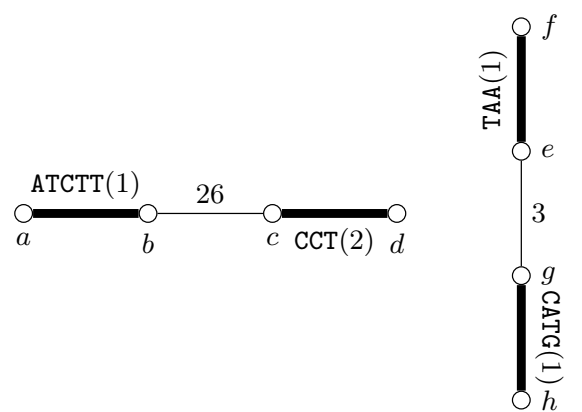

(d) Direct linearization from the scaffold graph. Directly searching two maximum weighted alternating paths such that the solution graph does not contain ambiguity yields a chimeric sequence TTA. .CATG (note that the sequence of $(f, e)$ is the reverse complement of the sequence of $(e, f))$ corresponding to $(f, e, g, h)$.

Fig. 3: Example for a hypothetical genome consisting of the chromosomes ATCTT . .CCT . . TAA and CCT . . CATG: a scaffold graph (Figure 3a), a solution graph (Figure 3b), scaffolds after solving SEMI-BRUTAL Cut (Figure 3c), and a direct linearization leading to chimeric solution (Figure 3d). 
Rule 1 Let $(u, \ldots, v)$ be a $\mu$-uniform alternating path in $G^{*}$ such that $\operatorname{deg}_{G^{*}}(u)=1$. Let $v w$ be a nonmatching edge. Then, create the $m(v w)$-uniform alternating path $\left(u^{\prime}, \ldots, v^{\prime}\right)$, create the edge $v^{\prime} w$ with multiplicity $m(v w)$, remove $v w$, and decrease the

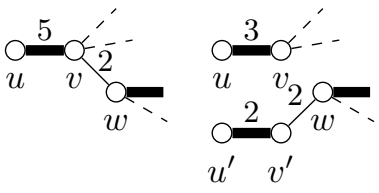
multiplicity of $(u, \ldots, v)$ by $m(v w)$.

We prove the correctness of this rule, that is, the input solution graph can be uniquely deconstructed if and only if the output solution graph can.

Proof. Let $G_{1}^{*}$ be a solution graph and $G_{2}^{*}$ be the solution resulting of the application of Rule 1 in $G_{1}^{*}$. Consider the function $\tau$ mapping multisets $\mathcal{W}$ of walks making up $G_{1}^{*}$ to multisets of walks in $G_{2}^{*}$. It works by replacing $(u, \ldots, v, w)$ (or $(w, v, \ldots, u))$ in $m(v w)$ walks by $\left(u^{\prime}, \ldots, v^{\prime}, w\right)$ (or $\left.\left(w, v^{\prime}, \ldots, u^{\prime}\right)\right)$. Clearly, no two different multisets for $G_{1}^{*}$ map to the same multiset for $G_{2}^{*}$ and, thus, $\tau$ is injective. To show that $\tau$ is surjective, suppose that there is a multiset $\mathcal{W}^{\prime}$ of walks making up $G_{2}^{*}$ that is not in the image of $\tau$. Note that any walk $W^{\prime}$ of $\mathcal{W}^{\prime}$ containing $\left(u^{\prime}, \ldots, v^{\prime}\right)$ also contains $\left(u^{\prime}, \ldots, v^{\prime}, w\right)$ (or $\left.\left(w, v^{\prime}, \ldots, u^{\prime}\right)\right)$ as a sub-walk, as for any edge $e$ in $\left(u^{\prime}, \ldots, v^{\prime}\right), m(e)=m\left(v^{\prime} w\right)$ and no walk starts with a non-matching edge. Thus, replacing $\left(u^{\prime}, \ldots, v^{\prime}, w\right)$ (or $\left.\left(w, v^{\prime}, \ldots, u^{\prime}\right)\right)$ by $(u, \ldots, v, w)$ (or $(w, v, \ldots, u))$ in all walks of $W^{\prime}$ yields a multiset $W$ of walks making up $G_{1}^{*}$ and $\tau(W)=W^{\prime}$. Thus, $\tau$ is a bijection implying that the number of different multisets of walks making up $G_{1}^{*}$ is equal to the number of multisets making up $G_{2}^{*}$.

Theorem 1. Let $G^{*}$ be a solution graph. Then, $G^{*}$ is made up of a unique multiset of alternating walks if and only if $G^{*}$ does not contain ambiguous paths or cycles.

Proof. " $\Rightarrow$ ": Suppose that $G^{*}$ contains an ambiguous cycle $c$. Let $\mu^{\prime}>1 \in \mathbb{N}$ such that $c$ is a $\mu^{\prime}$-uniform alternating cycle. For each $k \in \mathbb{N}$, let $c^{k}$ be the closed alternating walk which passes $k$ times across the edges of $c$. The two multisets of walks $\left\{c^{\mu^{\prime}}\right\}$ and $\left\{c^{1}, c^{\mu^{\prime}-1}\right\}$ make up $c$, contradicting the uniqueness of such a multiset. Suppose that $G^{*}$ contains an ambiguous path $p=(v, w, \ldots, x, y)$ and let $\mathcal{W}$ be a multiset of walks that make up $G^{*}$. Let $m \in \mathbb{N}$ such that $p$ is $\mu$-uniform and let $u v$ and $y z$ be non-matching edges incident to $v$ and $y$, respectively, whose respective multiplicities are strictly less than $m$. Thus, $\mu>1$. Note that no walk $W$ of $\mathcal{W}$ starts or ends with an inner edge $e \in M^{*}$ of $p$, since otherwise, $e$ is incident with a non-matching edge of $p$ that is traversed strictly less than $\mu$ times, as no walk of $\mathcal{W}$ starts or ends with a non-matching edge. Thus, each time a walk of $\mathcal{W}$ traverses $v w$, it also traverses $p$. Consider the graph $G_{\mathcal{W}}^{*}$ on the vertex set $\left\{\left(W_{1}, W_{2}\right) \mid\left(W_{1}, p, W_{2}\right) \in \mathcal{W}\right\}$ and $G_{\mathcal{W}}^{*}$ contains an edge $\left\{\left(W_{1}, W_{2}\right),\left(W_{1}^{\prime}, W_{2}^{\prime}\right)\right\}$ if and only if $W_{1}=W_{1}^{\prime}$ ("blue edge") or $W_{2}=W_{2}^{\prime}$ ("red edge"). Note that subwalks can be empty and no edge is blue and red at the same time, as otherwise, its endpoints are equal (but there are no self-loops in $G_{\mathcal{W}}^{*}$ ). Also note that the blue edges form a transitive subgraph of $G_{\mathcal{W}}^{*}$ and, by symmetry, so do the red edges. Since the multiplicity of $u v$ in $G^{*}$ is non-zero and different from that of $v w$, we 
know that $G_{\mathcal{W}}^{*}$ does not entirely consist of blue edges and, by symmetry, the same can be said for red edges. Thus, $G_{\mathcal{W}}^{*}$ is not a clique and, therefore, there are pairs $\left(W_{1}, W_{2}\right)$ and $\left(W_{1}^{\prime}, W_{2}^{\prime}\right)$ such that (a) $W=\left(W_{1}, p, W_{2}\right)$ and $W^{\prime}=\left(W_{1}^{\prime}, p, W_{2}^{\prime}\right)$ are (not necessarily distinct) walks in $\mathcal{W}$ and (b) $W_{1} \neq W_{1}^{\prime}$ and $W_{2} \neq W_{2}^{\prime}$. If $W \neq$ $W^{\prime}$, then the result of removing $W$ and $W^{\prime}$ from $\mathcal{W}$ and inserting $\left(W_{1}, p, W_{2}^{\prime}\right)$ and $\left(W_{1}^{\prime}, p, W_{2}\right)$ is another multiset of walks making up $G^{*}$. Thus, $W^{\prime}=W=$ $\left(X_{1}, p, X_{2}, p, X_{3}\right)$ for walks $X_{1}, X_{2}, X_{3}$ in $G^{*}$. But then, the result of removing $W$ from $\mathcal{W}$ and inserting $\left(X_{1}, p, X_{3}\right)$ and the closed walk consisting of $p$ and $X_{2}$ is another multiset of walks making up $G^{*}$. In both cases, $\mathcal{W}$ is not unique.

" $\Leftarrow$ ": Let $G^{*}$ be free of ambiguous paths or cycles. We suppose that Rule 1 is applied on $G^{*}$. If $G^{*}$ is empty, then $G^{*}$ has a unique multiset of walks making it up. If $G^{*}$ contains a uniform alternating cycle $c$, then since $c$ is not ambiguous, $c$ is 1-uniform. Hence, the unique multiset making up $c$ is $\{c\}$. Otherwise, let $\mu \in \mathbb{N}$ and let $p=(u, \ldots, v)$ be a maximal, $\mu$-uniform, alternating path in $G^{*}$ (as $p$ may consist of a single edge and $G^{*}$ is not empty, $p$ exists). Note that all inner vertices of $p$ have degree two in $G^{*}$ and suppose without loss of generality that $\operatorname{deg}_{G S}(u) \leq \operatorname{deg}_{G^{*}}(v)$. If $\operatorname{deg}_{G^{*}}(u)=1$ and $\operatorname{deg}_{G^{*}}(v) \geq 2$, then Rule 1 applies. Thus, suppose $\operatorname{deg}_{G^{*}}(u)>1$ and $\operatorname{deg}_{G^{*}}(v)>1$. Then, by maximality of $p$, both $u$ and $v$ are incident to a non-matching edge with multiplicity strictly less than $m$ and, thus, $p$ is ambiguous, contradicting the assumption that $G^{*}$ is free of ambiguous paths. Hence, $\operatorname{deg}_{G^{*}}(u)=1$ and $\operatorname{deg}_{G^{*}}(v)=1$, and $p$ is isolated. The multiset consisting of $\mu(u, \ldots, v)$-walks is the unique multiset making up $p$.

\section{Reduction rules}

In this section, we present a set of reduction rules that simplify instances of Semi-Brutal Cut. First, let us deal with some trivial cases to remove them from consideration.

Rule 2 Let $c$ be an isolated, $\mu$-uniform cycle in $\left(G^{*}, M^{*}, \omega, m\right)$. If $\mu=1$, then remove $c$. Otherwise, cut a vertex incident to the lightest non-matching edge of $c$.

Rule 3 Remove all isolated, uniform, alternating paths from $\left(G^{*}, M^{*}, \omega, m\right)$.

Rule 4 Let $u v \in M^{*}$ be a matching edge that does not occur in ambiguous paths and let $u$ and $v$ have degree at least two. Then, remove $u v$, add new vertices $u^{\prime}$ and $v^{\prime}$ and add the matching edges $u v^{\prime}$ and $v u^{\prime}$ with multiplicity $m(u v)$.

Correctness of Rule 4 follows immediately from the fact that no ambiguous path is changed, created or destroyed by applying the rule. Furthermore, since both $u^{\prime}$ and $v^{\prime}$ have degree one in the result $G^{\prime}$ of applying the rule, all solutions $X$ for $G^{\prime}$ avoid them and, since all scoring functions only depend on the non-matching edges incident to the solution, all solutions maintain their scores. 
Rule 5 Let $\mu \in \mathbb{N}$ and let $p=(u, \ldots, v)$ be $a$ $\mu$-uniform, alternating path in $G^{*}$. If uv is a nonmatching edge of $G^{*}$, then create two matching edges $u_{1} u_{2}$ and $v_{1} v_{2}$ with multiplicity $m(u v)$, add the nonmatching edges $u u_{1}$ and $v v_{1}$ with weight 0 and multiplicity $m(u v)$, and remove uv (and, for the weight score, decrease $k$ by $\omega(u v))$. In any case, remove all inner vertices of $p$ and create a matching edge uv

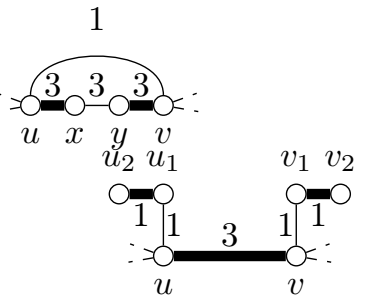
with multiplicity $\mu$.

Proof (Correctness of Rule 5). First, since no inner vertex of $p$ can be cut, replacing $p$ by a single matching edge does not change the score (cut- or weight) of a solution. It remains to show correctness for the case that $u v$ exists in $G^{*}$. Then $m(u v)<\mu$ since the input graph does not contain isolated cycles and, thus, $p$ is ambiguous. Thus, any solution $X$ for $G^{*}$ contains $u$ or $v$. In the output graph, $p$ is still ambiguous and either $u_{1} u$ or $v_{1} v$ must be removed in any solution. Further, $u_{1}$ and $v_{1}$ can be replaced by $u$ and $v$, respectively, in any solution for the output graph. Thus, $X$ is a solution in the input graph if and only if it is a solution in the output graph. $X$ has the same cut score in both input and output graph. Under the weight score, $\operatorname{score}(X)$ decreases by $\omega(u v)$.

Finally, note that all reduction rules can be applied in linear time. Further, it turns out that all matching edges of $G^{*}$ either occur in ambiguous paths or are incident with a degree-one vertex. In the latter case, we call the matching edge clean.

Proposition 1. Let $\left(G^{*}, M^{*}, \omega, m\right)$ be reduced with respect to the presented reduction rules. Then, it is free of ambiguous paths if and only if all its edges are clean.

Proof. " $\Rightarrow "$ : To show the contraposition, let $u v$ be an edge that is not clean, that is, $u$ has a neighbor $x \neq v$ and $v$ has a neighbor $r \neq u$. If $m(u x), m(v r)<$ $m(u v)$, then $u v$ is an ambiguous path, proving the claim. Otherwise, since $m(v x), m(u r) \leq m(u v)$ by definition of solution graph, $m(v x)=m(u v)$ or $m(u r)=m(u v)$. By symmetry, suppose that $m(v x)=m(u v)$ and let $y:=$ $M^{*}(x)$. By definition of solution graph, $m(x y) \geq m(v x)$. If $m(x y)=m(v x)$, then $p=(y, x, v, u)$ is an $m(u v)$-uniform, alternating path in $G^{*}$, contradicting reducedness with respect to Rule 5 . Thus, suppose $m(x y)>m(v x)$. But then, any ambiguous path containing $u v$ must end at $v$ and, since $v$ is not incident to an edge of multiplicity strictly less than $m(u v)$, we know that $u v$ is not contained in any ambiguous paths, contradicting reducedness with respect to Rule 4.

"६": To show the contraposition, let $p=(u, \ldots, v)$ be an ambiguous path in $G^{*}$ and note that both $u$ and $v$ are incident to a non-matching edge. But then, clearly, $u M^{*}(u)$ cannot be clean in $G^{*}$.

Given Proposition 1, the multiplicity function is no longer important since the presence of a vertex of degree one in a matching edge suffices to determine if the matching edge is ambiguous. SEMI-BRUTAL Cut can be now described as follows. 


\section{Semi-Brutal Cut (SBC)}

Input: A solution graph $\left(G^{*}, M^{*}, \omega\right)$ and some $k \in \mathbb{N}$.

Question: Is there a set $X$ of cuts of $G^{*}$ which makes all the matching edge clean and the score of $X$ is at most $k$ ?

\section{Polynomial cases}

In the following, we consider special solution graphs for which SEMI-BRUTAL CUT can be solved in polynomial time for all of the presented scoring functions. Recall that the goal is to clean all matching edges in $G^{*}$ (see Proposition 1).

\section{$5.1 \quad$ Sparse graphs}

We first show that SBC is polynomial for both scores into some classes of sparse graphs. First, we consider the class of trees. We suppose that the tree $G^{*}$ is rooted in an extremity of an ambiguous edge. Under the weight score, we can thus formulate the following dynamic program.

Let $x$ be a vertex, $T_{x}$ is the subgraph induced by the subtree rooted at $x$ and $M^{*}(x)$. For any vertex $x$, a table entry $c(x)$ represents the minimum score of a solution below $x$ in which $x$ has degree one in $T_{x}$ and $\bar{c}(x)$ represents the minimum score of a solution below $x$ in which $M^{*}(x)$ has degree one in $T_{x}$. For convenience, we set $\omega(e)=0$ for all matching edge $e$. If $x$ is a leaf of $G^{*}$ then, clearly, $c(x)=\bar{c}(x)=0$. For any non-leaf $x$, we set

$$
\begin{aligned}
& c(x)=\sum_{y \in \text { Children }(x)} \min (\bar{c}(y), c(y))+\omega(x y) \\
& \bar{c}(x)=\sum_{y \in \text { Children }(x)} \begin{cases}c(y) & \text { if } y=M^{*}(x) \\
\min (\bar{c}(y), c(y)+\omega(x y)) & \text { otherwise }\end{cases}
\end{aligned}
$$

Lemma 1. Those costs $c(x)$ and $\bar{c}(x)$ represent respectively the minimum weight score of a semi-brutal cut in the subtree rooted at $x$ when $x$ or $M^{*}(x)$ has degree one in $T_{x}$.

Notice that it may hapend that both extremities of a matching edge have degree one in an optimal solution, and in this case, we have $c(x)=\bar{c}(x)$.

Proof. We prove it by induction on the subtree's height. Let $x$ be any vertex in the tree. Let $h(x)$ denote the height of the subtree rooted at $x$. When $h(x)=0$, $x$ is a leaf and a solution of SEMI-BRUTAL CUT in $T_{x}$ consists in cutting nothing, thus the cost is zero. Suppose now that for any vertex $x^{\prime}$ with height $h\left(x^{\prime}\right)<h(x)$, $c\left(x^{\prime}\right)$ and $\bar{c}\left(x^{\prime}\right)$ satisfy the lemma's property. We prove that:

1. any solution $X$ of Semi-Brutal Cut in $T_{x}$ has $\operatorname{score}(X) \geq c(x)$ if $x$ has degree one in $T_{x}$ after applying $X$, or $\operatorname{score}(X) \geq \bar{c}(x)$ if $M^{*}(x)$ has degree one in $T_{x}$, and 
2. there exists a solution $S_{x}$ reaching the costs $c(x)$ and $\bar{c}(x)$.

1. Let $X$ be a solution of Semi-Brutal Cut in $T_{x}$. We denote by $X_{y}$ the restriction of $X$ to $T_{y}$, for any children $y$ of $x . X_{y}$ is trivially a solution of Semi-Brutal Cut in $T_{y}$, whose height is strictly less than $h(x)$. By induction hypothesis, $\operatorname{score}\left(X_{y}\right) \geq c(y)$ if all non-matching edges incident to $y$ in $T_{y}$ are removed in $X_{y}$, or $\operatorname{score}\left(X_{y}\right) \geq \bar{c}(y)$ otherwise.

- Suppose that all non-matching edges incident to $x$ in $T_{x}$ are removed in $X$. Thus, the weight score of $X$ contains the total weight of these nonmatching edges plus the score of any subsolution $X_{y}$ :

$$
\operatorname{score}(X)=\sum_{y \in \operatorname{Children}(x)\}} \operatorname{score}\left(X_{y}\right)+\omega(x y)
$$

Using the previous equation and induction hypothesis, we have $\operatorname{score}(X) \geq$ $c(x)$.

- Suppose that all non-matching edges incident to $M^{*}(x)$ in $T_{x}$ are removed after applying $X$. For any child $y$, the weight score of $X$ contains the score of $X_{y}$ plus $\omega(x y)$ if $y$ belongs to $X_{y}$ :

$$
\operatorname{score}(X)=\sum_{y \in \text { Children }(x)} \begin{cases}\operatorname{score}\left(X_{y}\right)+\omega(x y) & \text { if } y \in X_{y} \\ \operatorname{score}\left(X_{y}\right) & \text { otherwise }\end{cases}
$$

We distinguish $M^{*}(x)$ amongst children of $x$.

- If $M^{*}(x)$ is in Children $(x)$, then necessarily all incident edges to it has to be removed. In this case, $\operatorname{score}\left(X_{M^{*}(x)}\right) \geq c\left(M^{*}(x)\right)$, by induction hypothesis.

- For any other children $y$ of $x$, either $y$ has degree one in $X_{y}$, yielding a cost $c(y)+\omega(x y)$, or $M^{*}(y)$ has degree one in $X_{y}$, yielding a cost $\bar{c}(y)$. Hence, using the previous equation and induction hypothesis, we have $\operatorname{score}(X) \geq \bar{c}(x)$.

2. Now we show that is possible to build two solutions $X_{x}$ and $\bar{X}_{x}$ of SEMIBrutal Cut in $T_{x}$ with weight $c(x)$ and $\bar{c}(x)$, respectively. Considering a child $y$ of $x$, we denote by $X_{y}$ a solution of SEmi-Brutal Cut in $T_{y}$, where all non-matching edge incident to $y$ in $T_{y}$ are remoed, with weight score $c(y)$, and $\bar{X}_{y}$ a solution of SEMI-BRUTAL CUT in $T_{y}$, where all non-matching edge incident to $M^{*}(y)$ are removed in $T_{y}$, with weight score $\bar{c}(y)$. Such solutions do exist, by induction hypothesis.

- We define the set $X_{x}$ by:

$$
X_{x}=\{x\} \cup \bigcup_{y \in \text { Children }(x)} \begin{cases}X_{y} & \text { if } c(y)<\bar{c}(y) \\ \bar{X}_{y} & \text { otherwise. }\end{cases}
$$

Since $X_{y}$ and $\bar{X}_{y}$ are solutions of Semi-Brutal Cut in $T_{y}$, they clean all ambiguous edges below $y$. Removing all non-matching edges incident to $x$ cleans the ambiguous edge $x M^{*}(x)$ in $T_{x}$. Thus, $X_{x}$ is a solution of Semi-Brutal Cut in $T_{x}$, with weight score $c(x)$. 

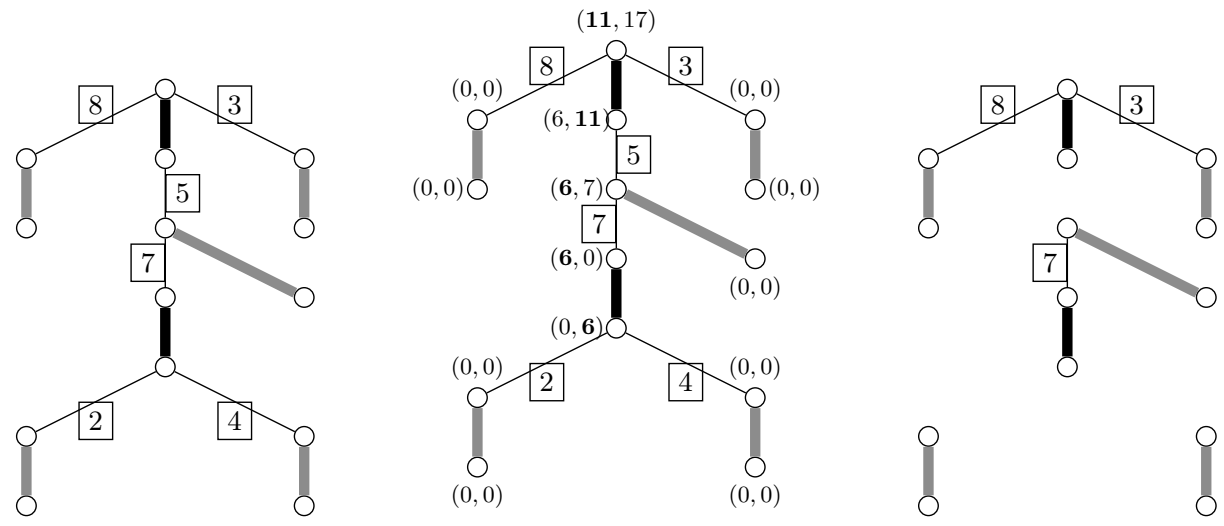

Fig. 4: Example of an application of the dynamic programming algorithm with matching edges (bold, gray if already clean) and weights (numbers in boxes). Left: the input solution graph $G^{*}$. Middle: $\operatorname{costs}(\bar{c}, c)$ after the bottom-up step. Bold figures indicate the backtracking path. For example, the minimal cost at the root is a non-cutting cost 11, which comes from the cutting cost 11 of its matching-child and non-cutting costs or cutting costs of non-matching children. Right: resulting solution graph after the backtracking step, which is made up of six paths.

- We define the set $\bar{X}_{x}$ by:

$$
\bar{X}_{x}=\bigcup_{y \in \operatorname{Children}(x)} \begin{cases}X_{y} & \text { if } c(y)+\omega(x y) \leq \bar{c}(y) \text { or } y=M^{*}(x) \\ \bar{X}_{y} & \text { otherwise }\end{cases}
$$

Since the $X_{y}$ and $\bar{X}_{y}$ are solutions of Semi-Brutal Cut in $T_{y}$, they clean all ambiguous edges below $y$. If $M^{*}(x)$ is below $x$, a solution removing all non-matching edges incident to $M^{*}(x)$ in $T_{M^{*}(x)}$ cleans $x M^{*}(x)$. For any other children of $x$, either $y$ belongs to $X_{x}$ or $M^{*}(y)$ has degree one, thus $X_{x}$ cleans $y M^{*}(y)$ in $T_{x}$. Thus $\bar{X}_{x}$ is a solution of SEMIBrutal CUT in $T_{x}$, with weight score $\bar{c}$.

Corollary 1. Using a bottom-up step computing these costs, setting the score of the root as the minimum between $c(r)$ and $\bar{c}(r)$ and backtracking those costs to decide which vertices should be cut leads to an optimal solution in linear time and space.

An example of the application of this dynamic program can be found in Figure 4 . While presented here for the weight score, we remark that this dynamic program can be modified to work for the cut score. For that we add a third table entry representing the fact $x$ is not cut and all neighbors of $x$ except $M^{*}(x)$ are in the solution. Hence, the formulation of the dynamic program is the following with 
$n(x)=0$ if $x$ is a leaf of $G^{*}$.

$$
\begin{aligned}
& c(x)=\sum_{y \in \text { Children }(x)} \min (\bar{c}(y), c(y), n(y))+1 \\
& \bar{c}(x)=\sum_{y \in \text { Children }(x)} \begin{cases}\min (c(y), n(y)) & \text { if } y=M^{*}(x) \\
\min (\bar{c}(y), c(y)) & \text { otherwise }\end{cases} \\
& n(x)=\sum_{y \in \text { Children }(x)} \begin{cases}\bar{c}(y) & \text { if } y=M^{*}(x) \\
c(y) & \text { otherwise }\end{cases}
\end{aligned}
$$

Since we can easily adapt the proof of Lemma 1 to the cut score formulation, we let the reader check the correctness of this dynamic program.

Theorem 2. On trees, Semi-Brutal Cut can be solved in linear time and space for both scoring functions.

As a side note, we remark that Semi-Brutal Cut can be solved in linear time if $\Delta\left(G^{*}\right)=2$. To this end, we just need to check the two possibilities of removing every second non-matching edge in every cycle. Since each cycle can be worked on individually and independently, this can be done in linear time. What remains can be solved in linear time with Theorem 2 .

Theorem 3. SEMI-BRUtal CUT can be solved in linear time on a collection of paths and cycles $\left(\Delta\left(G^{*}\right)=2\right)$ under both scores.

\subsection{Dense graphs}

In some classes of dense graphs, we can show that $\mathrm{SBC}$ is polynomial under the cut score. Concerning the weight score, Corollary 2 states that $\mathrm{SBC}$ is $\mathcal{N} \mathcal{P}$ complete for most dense classes (Section 6.1). For the following proofs, note that any graph can be solved with $\left|M^{*}\right|$ cuts by simply cutting an arbitrary extremity of all matching edges.

Theorem 4. Semi-Brutal Cut can be solved in linear time for cut score on complete bipartite graphs.

Proof. Note that, if $G^{*}$ is bipartite, both cells of the partition have equal size since $M^{*}$ is a bijection between the two. Let $K_{n, n}$ be a complete bipartite graph (with $n:=\left|M^{*}\right|$ and suppose that $n \geq 2$ as, otherwise, matching edges are already clean. Then, it is sufficient to cut all but one vertex of any of the two cells of the bipartition to turn all matching edges clean. To show that $n-1$ cuts are also necessary, assume that there is a solution $X$ with cut score $n-2$. Since there are $n$ matching edges in $G^{*}$, there are two matching edges $u v$ and $x y$ that do not intersect $X$. Since $G^{*}$ is complete bipartite, $(u, v, x, y)$ forms an alternating cycle in $G^{*}$, so neither $u v$ nor $x y$ are clean.

Theorem 5. Semi-Brutal Cut can be solved in linear time under cut score on complete graphs. 
Proof. If any solution does not contain a cut in a matching edge $u v$, then either all neighbors of $u$ or all neighbors $v$ are cut, which implies a solution with a cut score of $\left|V\left(G^{*}\right)\right|-2>\left|M^{*}\right|$. Hence, $\left|M^{*}\right|$ cuts are necessary.

Theorem 6. Semi-Brutal Cut can be solved in linear time under cut score on co-bipartite graphs. $^{7}$

Proof. In this proof, suppose that $\left|M^{*}\right|>2$ as, otherwise, the claim trivially holds. Let $\left(V_{1}, V_{2}\right)$ denote a bipartition of the vertices of $G^{*}$ into two cliques. First, assume that $\left(G^{*}, M^{*}, \omega\right)$ has a solution $X$ with $|X|=\left|M^{*}\right|-2$ cuts. Then, there are matching edges $u v$ and $x y$ that avoid $X$. If either $V_{1}$ or $V_{2}$ intersects $u v x y$ in at least three vertices, say $u, v$, and $x$, then $u v$ is not clean. Thus, uvxy intersects both cells in exactly two vertices. If one cell contains $u x$ and the other $v y$, then $u v x y$ induces a cycle, and neither $u v$ nor $x y$ are clean. Thus, without loss of generality, let $u v \subseteq V_{1}$ and $x y \subseteq V_{2}$. But then, all other vertices have to be cut, implying $|X| \geq 2\left(\left|M^{*}\right|-2\right)>\left|M^{*}\right|-2$.

Since we know that no solution $X$ with $|X| \leq\left|M^{*}\right|-2$ exists and a solution $X$ with $|X|=\left|M^{*}\right|$ is trivial, we just have to check if $G^{*}$ contains a matching edge $u v$ such that we can cut the vertices of $N(u)-v$ instead of cutting $u$ or $v$. We show that $G^{*}$ can be solved with $\left|M^{*}\right|-1$ cuts if and only if there is a matching edge $u v$ with $|N(u)| \leq\left|M^{*}\right|$ and there are no matching edges $x y \subseteq N(u)$. Since this can be checked in linear time, the theorem follows.

" $\Rightarrow$ ": If there is a solution $X$ for $G^{*}$ with $|X|=\left|M^{*}\right|-1$, then there is a matching edge $u v$ avoiding $X$ and all other matching edges intersect $X$ in exactly one extremity. By symmetry, let $N(u)-v \subseteq X$, implying $|N(u)| \leq\left|M^{*}\right|$ and, as each matching edge except $u v$ contains only a single cut, no matching edge $x y$ is included in $X$ and, thus, in $N(u)$.

" $\Leftarrow$ ": Let $Q$ be a set containing an arbitrary extremity of each $x y \in M^{*}$ with $x y \cap N(u)=\varnothing$ and let $X:=Q \cup N(u)-v$. Then, $|Q|=\left|M^{*}\right|-|N(u)|$ and $|X|=|Q|+|N(u)|-1=\left|M^{*}\right|-1$. Towards a contradiction, assume that $X$ is not a solution, that is, some matching edge $x y \in M^{*}$ is not clean. Then, $x y=u v$ since all other matching edges contain a cut. But $u v$ is clean since $N(u)-v \subseteq X$.

\section{Computational Hardness}

\subsection{Hardness in Sparse Graphs}

While SEmi-Brutal Cut is known to be $\mathcal{N} \mathcal{P}$-complete for both cut and weight score [?], we extend the cut-score hardness to planar, bipartite, subcubic graphs.

Theorem 7. Semi-Brutal Cut is $\mathcal{N} \mathcal{P}$-complete under both scores, even if the graph is planar, bipartite and subcubic.

\footnotetext{
${ }^{7}$ A graph is co-bipartite if its vertices can be partitioned into two cliques.
} 

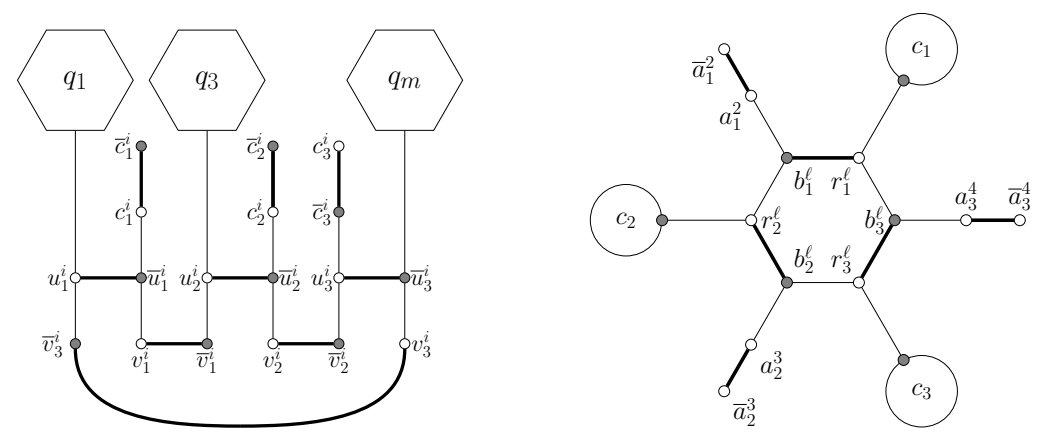

Fig. 5: Matching edges are bold. Left: variable gadget $c_{x_{i}}$ linked to the clause gadgets $q_{1}, q_{3}$ and $q_{m}, m \notin\{1,3\}$, where $x_{i}$ occurs positively in $C_{1}$ and $C_{3}$ and negatively in $C_{m}$. Right: clause gadget corresponding to the clause $C_{\ell}=\left(x_{1} \vee\right.$ $\left.\overline{x_{2}} \vee x_{3}\right)$.

To this end, we reduce the classic $\mathcal{N} \mathcal{P}$-complete 3 -SAT [? ] problem to SBC.

\section{3-SATisfiability (3-SAT)}

Input: A boolean formula $\varphi$ in conjunctive normal form where each clause contains exactly three literals.

Question: Is there a satisfying assignment $\beta$ for $\varphi$ ?

Construction 1 Let $\varphi$ be an instance of 3 -SAT with $n$ variables $x_{1}, \ldots, x_{n}$ and $m$ clauses $C_{1}, \ldots, C_{m}$. For each variable $x_{i}$, let $\psi_{i}$ be the list of indices $\ell$ such that $C_{\ell}$ contains $x_{i}$ and $\left|\psi_{i}\right|$ is the number of occurrences of $x_{i}$ in $\varphi$. We construct the following solution graph $\left(G^{*}, M^{*}, \omega\right)$ with a proper 2-coloring of $G^{*}$ (see Figure 5).

- For each $x_{i}$, we construct a cycle $c_{i}$ on the vertex set $\bigcup_{j \leq\left|\psi_{i}\right|}\left\{u_{j}^{i}, \bar{u}_{j}^{i}, v_{j}^{i}, \bar{v}_{j}^{i}\right\}$ such that, for all $j \leq\left|\psi_{i}\right|$,

- $u_{j}^{i} \bar{u}_{j}^{i}, v_{j}^{i} \bar{v}_{j}^{i} \in M^{*}$, and

- the vertices $u_{j}^{i}$ and $v_{j}^{i}$ are blue and the vertices $\bar{u}_{j}^{i}$ and $\bar{v}_{j}^{i}$ are red.

- For each $C_{\ell}$, we construct an alternating 6 -cycle $q_{\ell}$ on the vertex set $\bigcup_{j \leq 3}\left\{r_{j}^{\ell}, b_{j}^{\ell}\right\}$ such that, for all $j \leq 3,\left\{r_{j}^{\ell}, b_{j}^{\ell}\right\} \in M^{*}$, and $r_{j}^{\ell}$ is red and $b_{j}^{\ell}$ is blue.

- For each clause $C_{\ell}$ and each $j \leq 3$, let $x_{i}$ be the $j^{\text {th }}$ literal of $C_{\ell}$ and let $t$ be such that $C_{\ell}$ is the $t^{\text {th }}$ clause in which $x_{i}$ occurs. Then,

- create two singles matching edges $a_{t}^{i} \bar{a}_{t}^{i}$ and $c_{t}^{i} \bar{c}_{t}^{i}$, where $a_{t}^{i}$ and $c_{t}^{i}$ are blue and $\bar{a}_{t}^{i}$ and $\bar{c}_{t}^{i}$ are red,

- if $x_{i}$ is a positive literal, introduce the edges $r_{j}^{\ell} u_{t}^{i}, b_{j}^{\ell} \bar{a}_{t}^{i}$ and $\bar{u}_{t}^{i} c_{t}^{i}$, and

- if $x_{i}$ is a negative literal, introduce the edges $b_{j}^{\ell} \bar{u}_{t}^{i}, r_{j}^{\ell} a_{j}^{\ell}$ and $u_{t}^{i} \bar{c}_{t}^{i}$.

- Each non-matching edge has weight one, except the edges $\bar{u}_{t}^{i} c_{t}^{i}$ and $u_{t}^{i} \bar{c}_{t}^{i}$ which have weight zero.

Note that each matching edge except the $a_{i}^{\ell} \bar{a}_{i}^{\ell}$ and $c_{t}^{i} \bar{c}_{t}^{i}$ is ambiguous. Clearly, Construction 1 can be carried out in polynomial time. Further, the resulting 
graph $G^{*}$ is bipartite and $\Delta\left(G^{*}\right)=3$. We first use Construction 1 on a restricted subcase of 3-SAT defined below.

Monotone 3-Satisfiability (Monotone 3-SAT)

Input: A boolean formula $\varphi$ in conjunctive normal form where each clause contains exactly three positive literals or three negative literals.

Question: Is there a satisfying assignment $\beta$ for $\varphi$ ?

In order to prove Theorem 7, we use the following properties of Construction 1 , yielding a "canonical" set of cuts, if the input formula is monotone.

Lemma 2. Let $X \subseteq V\left(G^{*}\right)$ be a set of cuts cleaning all ambiguous edges in $\left(G^{*}, M^{*}, \omega\right)$, let $c_{i}$ be a variable gadget and let $q_{\ell}$ be a clause gadget. Let $s=1$ under the cut score and $s=2$ under the weight score. We suppose that we start by cutting the vertices in the variable gadgets, and then we cut the vertices in the clause gadgets. There is a set $X^{\prime}$ of cuts with score $\left(X^{\prime}\right) \leq \operatorname{score}(X)$ that also cleans all ambiguous edges and

(a) $\operatorname{score}\left(X^{\prime} \cap V\left(c_{i}\right)\right) \geq s\left|\psi_{i}\right|$ and score $\left(X^{\prime} \cap V\left(q_{\ell}\right)\right) \geq 2$,

(b) if score $\left(X^{\prime} \cap V\left(c_{i}\right)\right)=s\left|\psi_{i}\right|$, then $X^{\prime} \cap V\left(c_{i}\right)$ is either $\bigcup_{j \leq\left|\psi_{i}\right|}\left\{u_{j}^{i}\right\}$ or $\bigcup_{j \leq\left|\psi_{i}\right|}\left\{\bar{u}_{j}^{i}\right\}$ (in $X^{\prime}$, cuts are only on positive sides or only on negative sides), (c) score $\left(X^{\prime} \cap V\left(q_{\ell}\right)\right)=2$ if and only if $X^{\prime}$ contains a vertex adjacent to $q_{\ell}$ (the score is two in a clause gadget iff it has been isolated by a cut in an adjacent variable gadget, meaning that the variable satisfies the clause).

Proof. (a): For each $j \leq\left|\psi_{i}\right|$, we need to remove two edges to clean the ambiguous edges $\left\{u_{j}^{i}, \bar{u}_{j}^{i}\right\}$, which can be done only by cutting at least one vertex among $\left\{\bar{v}_{j-1}^{i}, u_{j}^{i}, \bar{u}_{j}^{i}, v_{j}^{i}\right\}$. Thus, we need to remove at least $2\left|\psi_{i}\right|$ edges with at least $\left|\psi_{i}\right|$ cuts, that is score $\left(X^{\prime} \cap V\left(c_{i}\right)\right) \geq s\left|\psi_{j}\right|$. In the clause $q_{\ell}$, we need to remove at least two edges in the inner cycles, which can be done by cutting at least two vertices. Thus, we have $\operatorname{score}\left(X^{\prime} \cap V\left(q_{\ell}\right)\right) \geq 2$.

(b): Note that cutting all vertices in either $\bigcup_{j \leq\left|\psi_{i}\right|}\left\{u_{j}^{i}\right\}$ or $\bigcup_{j \leq\left|\psi_{i}\right|}\left\{\bar{u}_{j}^{i}\right\}$ suffices to remove all ambiguous path in $c_{i}$. In that case, we have $\operatorname{score}\left(X^{\prime} \cap V\left(c_{i}\right)\right)=s\left|\psi_{i}\right|$. If $X$ contains some $\bar{u}_{j}^{i}$ and does not contain $\bar{u}_{j+1}^{i}$ for some $j$, then we need a extra cut to linearize $\left\{v_{j}^{i}, \bar{v}_{j}^{i}\right\}$ (and analogously for $u_{j}^{i}$ ) which will increase the score by one. Hence, if $\left|X \cap V\left(c_{i}\right)\right|=s\left|\psi_{i}\right|$, we can suppose that $X$ contains either $\bigcup_{j \leq\left|\psi_{i}\right|}\left\{u_{j}^{i}\right\}$ or $\bigcup_{j \leq\left|\psi_{i}\right|}\left\{\bar{u}_{j}^{i}\right\}$. If $X$ contains a cut in some $v_{j}^{i}$ or some $\bar{v}_{j}^{i}$, then since the edge $\left\{v_{j}^{i}, \bar{v}_{j}^{i}\right\}$ is already clean by a cut in $\left\{\bar{u}_{j}^{i}, u_{j+1}^{i}\right\}$, we can can remove the cut in $X^{\prime}$. 
(c): We need to remove at least two non-zero weighted edges from the inner cycle of $C_{\ell}$. Suppose that all literals of $C_{\ell}$ occurs positively. Suppose by symmetry that $\left\{b_{1}^{\ell}, b_{2}^{\ell}\right\} \in$ $X^{\prime}$. If the leaving edge incident to $r_{3}^{\ell}$ is cut, then all ambiguous edges of $C_{\ell}$ are destroyed. Otherwise, we need to remove one more non-zero weighted edge from $q_{\ell}$ which must add another cut (see Figure 6).

We are now able to prove Theorem 7 .

Proof. of Theorem 7

Recall that MONOTONE 3-SAT remains $\mathcal{N} \mathcal{P}$-complete if the input formula is planar [?] and, in this case, since each gadget is planar and the edges between the clause gadget and the variable gadget can be placed in any order on the gadgets, the graph produced by Construction 1 can also be assumed to be planar. Since, clearly, Semi-Brutal Cut $\in \mathcal{N} \mathcal{P}$, it remains to show that Construction 1 is correct, that is $\varphi$ is satisfiable if and only if the solution graph $\left(G^{*}, M^{*}, \omega\right)$ resulting from Construction 1 can be linearized with a score of $(3 s+2) m$.

" $\Rightarrow$ ": Let $\beta$ be a satisfying assignment for $\varphi$. Then, for each variable $x_{i}$ and for all $j \leq\left|\psi_{i}\right|$, we cut the vertices $u_{j}^{i}$ if $\beta\left(x_{i}\right)=1$ and the vertices $\bar{u}_{j}^{i}$ otherwise. As $\beta$ is satisfying, this removes at least one edge adjacent to each clause gadget. Thus, according to Lemma 2(c), we can clean the matching edges in each clause gadget $q_{j}$ with a score of two. Since we also cut either the vertices $u_{j}^{i}$ or the vertices $\bar{u}_{j}^{i}$ for each vertex gadget, we conclude that all matching edges of the result are clean, and we have a score of $2 m+\sum_{i} s\left|\psi_{i}\right|=(2+3 s) m$.

" $\Leftarrow$ ": Let $X \subseteq V$ be the set of vertices such that cutting each vertex of $X$ destroys all ambiguous paths in $\left(G^{*}, M^{*}, \omega\right)$ and $\operatorname{score}(X)=(3 s+2) m$. According to Lemma 2(a), each variable gadget has a score of $s\left|\psi_{i}\right|$ and each clause gadget has a score of two. Moreover, by Lemma 2(b), for each variable gadget $c_{i}$, we can suppose that $X \cap V\left(c_{i}\right)$ equals $\bigcup_{j \leq\left|\psi_{i}\right|}\left\{u_{j}^{i}\right\}$ or $\bigcup_{j \leq\left|\psi_{i}\right|}\left\{\bar{u}_{j}^{i}\right\}$. In the former case, we set $\beta\left(x_{i}\right)=1$ and, in the latter, we set $\beta\left(x_{i}\right)=0$. To show that $\beta$ satisfies $\varphi$, assume that there is a clause $C_{\ell}$ that is not satisfied by $\beta$. Then, none of the edges incident to $q_{\ell}$ is cut which, by Lemma 2(c), contradicts the fact that the score of $q_{\ell}$ is equal to two.

\subsection{Hardness in Dense Graphs}

We can see that if we add some zero weighted non-matching edges on a solution graph, it does not change the weight score of an optimal solution. This observation leads to the following result.

Corollary 2. Let $\mathcal{G}$ be a class of graphs such that, for any planar, subcubic, bipartite graph $G, \mathcal{G}$ contains a supergraph of $G$. Then, Semi-Brutal Cut is $\mathcal{N P}$-complete on $\mathcal{G}$ under the weight score. 
Concerning the cut score, in some classes of dense graphs, SBC can be solved in polynomial time (see Section 5.2). However, we show in this part an example of a class of dense graphs where computing an optimal solution for SBC is $\mathcal{N} \mathcal{P}$-hard under the cut score. A graph $G$ is a split graph if we can partition its vertices into two sets $I$ and $C$ inducing an independent set and a clique, respectively. We show that SBC is hard to compute in split graphs by doing a reduction from the well-known VERTEX COVER problem, defined below.

Vertex COVER (VC)

Input: An undirected graph $G$ and a number $k \in \mathbb{N}$.

Question: Is there a $V^{\prime} \subseteq V(G)$ with $\left|V^{\prime}\right| \leq k$ intersecting all $e \in$ $E(G)$ ?

Construction 2 Let $G$ be an instance of VERTEX COVER, we suppose that $G$ is connected. We construct the following solution graph $G^{*}$ as follows:

1. for each vertex $v$ of $G$, construct a matching edge $v_{1} v_{2}$,

2. for each edge uv of $G$, add the non-matching edges $v_{1} u_{2}$ and $u_{1} v_{2}$, and

3. for each pair of vertices $(u, v)$, add the edge $u_{1} v_{1}$.

The set of $v_{1}$ (resp. $v_{2}$ ) vertices form a clique (resp. is independent). Thus, $G^{*}$ is a split graph. Note that all matching edges are ambiguous.
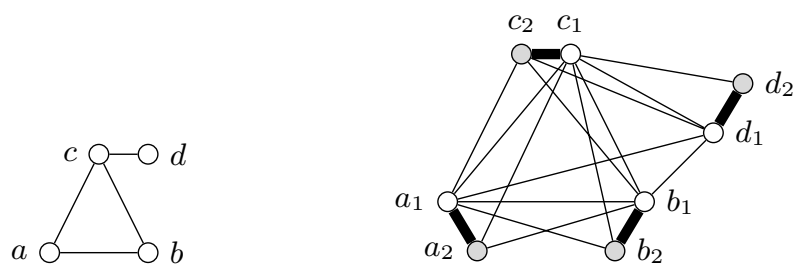

Fig. 7: Construction 2 transforms left instance into right instance, where gray vertices form an independent set and white vertices form a clique.

Theorem 8. Semi-Brutal Cut is $\mathcal{N} \mathcal{P}$-hard under the cut score on split graphs.

Proof. We show that $G$ has a size- $k$ vertex cover if and only if using $k$ cuts suffices to clean all matching edges in $G^{*}$.

" $\Rightarrow$ ": Let $V^{\prime}$ be a vertex cover of $G$. For each vertex $v \in V^{\prime}$, cut the vertex $v_{1}$ in $G^{*}$. Suppose that there is a matching edge $v_{1} v_{2}$ that is not clean. There is an edge $v_{2} u_{1}$ that is not removed by a cut. Then, neither of the two vertices $u$ and $v$ belong to $V^{\prime}$ and the edge $u v$ is not covered in $G$, contradicting the fact that $V^{\prime}$ is a vertex cover.

" $\Leftarrow$ ": Let $X$ be a solution of SBC under the cut score for $G^{*}$. For each $v \in V(G)$, suppose that $v_{2} \notin X$, since otherwise, $X^{\prime}=\left(X \backslash\left\{v_{2}\right\}\right) \cup\left\{v_{1}\right\}$ is also a solution and $\left|X^{\prime}\right| \leq|X|$. For each vertex $v_{1} \in X$, add the vertex $v$ in the vertex 
cover of $G$. If there is an edge $u v$ that is not covered, then $\left\{u_{1}, u_{2}, v_{1}, v_{2}\right\} \cup X=\varnothing$, and since $\left(u_{1}, u_{2}, v_{1}, v_{2}\right)$ is a cycle, the matching edges $u_{1} u_{2}$ and $v_{1} v_{2}$ are not clean.

Recall that VerTex Cover cannot be solved in $2^{o(n)}$ time unless $\mathrm{ETH}^{8}$ fails [?]. Since Construction 2 is linear on vertices and edges, we obtain the following result.

Corollary 3. There is no algorithm solving SEmi-Brutal CUT with cut score in $2^{o(n)}$ in split graphs.

\section{$7 \quad$ Non-Approximability}

In this section, we prove approximation lower bounds for Semi-Brutal Cut. First recall the definition of $L$-reduction between two hard problems $\Pi$ and $\Pi^{\prime}$, described by Papadimitriou and Yannakakis [? ]. This reduction consists of polynomial-time computable functions $f$ and $g$ such that, for each instance $x$ of $\Pi, f(x)$ is an instance of $\Pi^{\prime}$ and for each feasible solution $y^{\prime}$ for $f(x), g\left(y^{\prime}\right)$ is a feasible solution for $x$. Moreover, let $\Pi^{\prime \prime} \in\left\{\Pi, \Pi^{\prime}\right\}$, we denote by $O P T_{\Pi^{\prime \prime}}$ the value of an optimal solution of $\Pi^{\prime \prime}$ and by $\operatorname{val}_{\Pi^{\prime \prime}}\left(y^{\prime \prime}\right)$ the value of a solution $y^{\prime \prime}$ of an instance of $\Pi^{\prime \prime}$. There are constants $\alpha, \beta>0$ such that:

1. $O P T_{\Pi^{\prime}}(f(x)) \leq \alpha O P T_{\Pi}(x)$ and

2. $\left|\operatorname{val}_{\Pi}\left(g\left(y^{\prime}\right)\right)-O P T_{\Pi}(x)\right| \leq \beta\left|\operatorname{val}_{\Pi^{\prime}}\left(y^{\prime}\right)-O P T_{\Pi^{\prime}}(f(x))\right|$.

\subsection{Reduction from MAX 3-SAT(4)}

In the following, we present an $L$-reduction from the classical problem MAX 3-SAT(4) to Semi-Brutal Cut under the cut score.

\section{MAX 3-SAT(4)}

Input: A boolean formula $\varphi$ in exact 3-CNF where every variable occurs in four clauses.

Task: Find an assignment that satisfies a maximum number of clauses.

Our goal is to reuse Construction 1 to reduce MAX 3-SAT(4), such that each unsatisfied clause in $\phi$ causes an additional cut in $G^{*}$. Indeed, if there is no optimal solution with a score of $5 m$ in $G^{*}$ (that is, if $\varphi$ can not be satisfied), then we can spend an "extra" cut per unsatisfied clause to solve $G^{*}$. The inverse, however, does not hold if there is a variable $x_{i}$ that occurs two times positively and two times negatively. Indeed, by cutting five vertices in $C_{i}$, we may be able to satisfy the four clauses where $x_{i}$ occurs (see Figure 8). Thus, in the following, we modify Construction 1 slightly.

\footnotetext{
8 The (widely believed) "Exponential Time Hypothesis" (ETH) states that the boolean satisfyability problem (SAT) cannot be solved in $2^{o(n)}$ time, where $n$ is the number of variables of the input formula.
} 

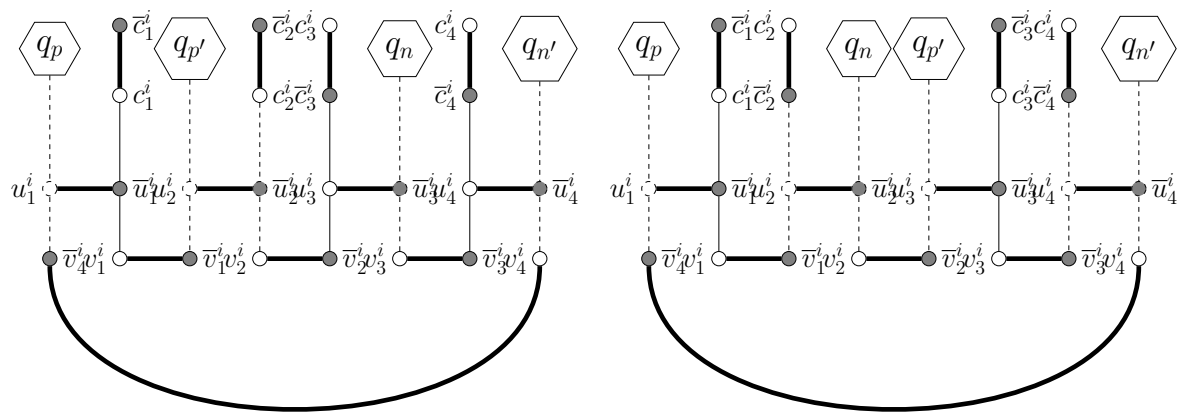

Fig. 8: Matching edges are bold. Example of variable gadget $r_{i}$ which occurs two times positively and two times negatively in Construction 1 (Left) and Construction 3 (Right). The cut-vertices are dashed. We can see that we need to add a cut in Construction 3 in order to remove all the edges leaving the gadget.

Construction 3 We reuse Construction 1 and change some variable gadgets. Let $x_{i}$ be a variable which two times positively and two times negatively. Before building the gadget $c_{i}$, we modify the clauses order in $\psi_{i}$ by interleaving positive and negative clauses. Other variable gadgets remain unchanged.

The resulting graph $G^{*}$ is bipartite and subcubic. An example of a variable gadget defined in Construction 3 is given in Figure 8. Notice that, if we do not take in account the weight on the edges, all clauses are symmetric. Thus, the properties (a) and (c) of Lemma 2 hold. We can add the following property:

Lemma 3. Let $X \subseteq V\left(G^{*}\right)$ be an optimal set of cuts that cleans all matching edges in $\left(G^{*}, M^{*}, \omega\right)$, let $c_{i}$ be a variable gadget. There is a set $X^{\prime}$ of cuts with score $\left(X^{\prime}\right)=\operatorname{score}(X)$ that also clean all matching edges, and $X^{\prime} \cap V\left(c_{i}\right)$ is either $\bigcup_{j \leq\left|\psi_{i}\right|}\left\{u_{j}^{i}\right\}$ or $\bigcup_{j \leq\left|\psi_{i}\right|}\left\{\bar{u}_{j}^{i}\right\}$.

Proof. Recall that $X$ covers the edges of $M^{*}$ and, by Lemma 2(a), score $(X \cap$ $\left.V\left(c_{i}\right)\right) \geq\left|\psi_{i}\right|$. By symmetry, suppose that $x_{i}$ occurs mostly positively in $\varphi$. If $x_{i}$ occurs four times positively, then replacing $X \cap V\left(c_{i}\right)$ by $\bigcup_{j \leq\left|\psi_{i}\right|}\left\{u_{j}^{i}\right\}$ in $X$ yields a solution $X^{\prime}$ as sought. Thus, suppose that $x_{i}$ occurs three times positively. Let $C_{\ell}$ be the clause where $x_{i}$ occurs negatively and let $z$ denote the neighbor of $\bar{u}_{j}^{i}$ in $c_{\ell}$. If $\operatorname{score}\left(X \cap V\left(c_{i}\right)\right)>\left|\psi_{i}\right|$, then replacing $X \cap c_{i}$ by $\bigcup_{j \leq\left|\psi_{i}\right|}\left\{u_{j}^{i}\right\}$ plus $z$ yields a solution $X^{\prime}$ as sought. Finally, if score $\left(X \cap V\left(c_{i}\right)\right)=\left|\psi_{i}\right|$, then $X$ already corresponds to $X^{\prime}$ as, otherwise, some ambiguous edge $v_{j}^{i} \bar{v}_{j}^{i}$ is not clean.

Suppose now that $x_{i}$ occurs two times positively and two times negatively. Note that one cut in $r_{i^{\prime}}$ is not enough to clean all ambiguous edges and cutting either the vertices $\left\{u_{1}^{i^{\prime}}, u_{2}^{i^{\prime}}\right\}$ or the vertices $\left\{\bar{u}_{1}^{i^{\prime}}, \bar{u}_{2}^{i^{\prime}}\right\}$ cleans all matching edges in the variable gadget. Further if $X$ cuts $\left\{v_{1}^{i^{\prime}}, v_{2}^{i^{\prime}}\right\}$ or $\left\{\bar{v}_{1}^{i^{\prime}}, \bar{v}_{2}^{i^{\prime}}\right\}$, then we can instead cut $\left\{u_{1}^{i^{\prime}}, u_{2}^{i^{\prime}}\right\}$ or $\left\{\bar{u}_{1}^{i^{\prime}}, \bar{u}_{2}^{i^{\prime}}\right\}$, respectively, without creating ambiguous edges. Suppose without loss of generality that $\left\{u_{1}^{i^{\prime}}, u_{2}^{i^{\prime}}\right\} \subseteq X$. Suppose further that there is 
some $\bar{u} \in X \cap V\left(r_{i^{\prime}}\right) \backslash\left\{u_{1}^{i^{\prime}}, u_{2}^{i^{\prime}}\right\}$. Then, there is some clause gadget $q_{n}$ linked to $\bar{u}$ since, otherwise, $X \backslash\{\bar{u}\}$ is also a solution, contradicting optimality of $X$. Since all matching edges of $r_{i^{\prime}}$ are already clean, the cut can only remove the edge between $\bar{u}$ and $q_{n}$. Let $z$ be the neighbor of $\bar{u}$ in $q_{n}$. In $X$, the two non-matching edges incident to $z$ must be removed, otherwise it contradicts the optimality of $X$. Thus, we can replace $\bar{u}$ by its neighbor in $q_{n}$ without changing the score of $X$. By swapping the one or two cuts in $X \cap V\left(r_{i^{\prime}}\right) \backslash\left\{u_{1}^{i^{\prime}}, u_{2}^{i^{\prime}}\right\}$, we obtain $X^{\prime} \cap V\left(r_{j}\right)=\left\{u_{1}^{i^{\prime}}, u_{2}^{i^{\prime}}\right\}$.

Theorem 9. It is NP-hard to approximate Semi-Brutal Cut to any factor better than $1+\frac{7\left(\epsilon_{4}-1\right)}{41 \cdot \epsilon_{4}}$ under the cut score, even on subcubic bipartite graphs.

Proof. First, note that it is $\mathcal{N} \mathcal{P}$-hard to approximate MAx 3-SAT(4) to any factor $\epsilon_{4} \leq 1.00052$, unless $\mathcal{P}=\mathcal{N} \mathcal{P}$ [? ]. Recall that in an optimal solution of MAX 3-SAT(4), at least $7 / 8$ of the clauses are satisfied [? ], yielding

$$
\operatorname{OPT}(\varphi) \geq 7 \mathrm{~m} / 8 \text {. }
$$

To show that Construction 3 constitutes an $L$-reduction, let $f$ be a function transforming any instance $\varphi$ of MAX 3-SAT(4) into an instance $I$ of SEMIBrutal Cut as above, let $X$ be a feasible solution for $I$ corresponding to the properties of Lemma 2(a), Lemma 2(c) and Lemma 3, and let $g$ be the function that transforms $X$ into an assignment as constructed in the proof of Theorem 7: each variable $x_{i}$ is set to true if $X$ cuts $u_{j}^{i}$ for all $j$, and false, otherwise. By Lemma 3 , for each clause gadget $q_{\ell}$ without an adjacent vertex in $X$, the "extra" cut occurs in $q_{\ell}$. Hence, we can linearize $I$ with one more cut for each of the at most $m / 8$ unsatisfied clauses in $\varphi$. Thus,

$$
O P T(I) \leq 5 m+m / 8 \stackrel{(1)}{\leq} 41 / 7 O P T(\varphi)
$$

An important obstacle to overcome (and reason why Construction 1 is not enough for Theorem 9) is that an approximate solution to SBC might spend extra cuts in variable gadgets in order to "change the assignment" of a variable $x_{i}$ mid-way. However, since each variable occurs at most four times, this only happens for variables that occur two times positively and two times negatively. Now, with our modification to Construction 1 and by Lemma 3, we can observe that each extra cut occurs in an unsatisfied clause gadget. Thus, the number of satisfied clauses of $\varphi$ and the clause gadgets in which we have to spend extra cuts add up to $m$. Hence,

$$
6 m=\operatorname{val}(g(X))+\operatorname{val}(X)=O P T(I)+O P T(\varphi)
$$

Thus, we constructed an $L$-reduction with $\alpha=41 / 7, \beta=1$ and, since $\epsilon_{4}$. $\operatorname{val}(g(X)) \leq O P T(\varphi)$, we conclude

$$
\begin{aligned}
\operatorname{val}(X) & \stackrel{(3)}{=} O P T(I)+O P T(\varphi)-\operatorname{val}(g(X)) \\
& \geq O P T(I)+\left(1-1 / \epsilon_{4}\right) \cdot O P T(I) \\
& \stackrel{(2)}{\geq}\left(1+\frac{7\left(\epsilon_{4}-1\right)}{41 \cdot \epsilon_{4}}\right) \cdot O P T(I)
\end{aligned}
$$




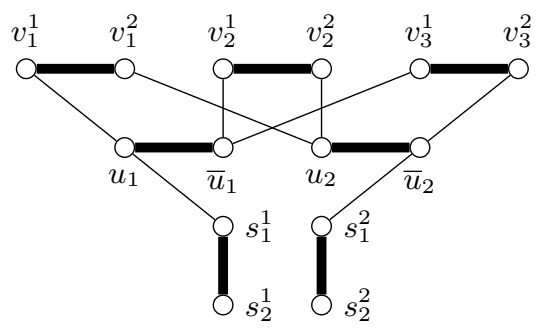

Fig. 9: The graph produced by Construction 4 and on input $\varphi=\left(x_{1} \vee x_{2}\right) \wedge$ $\left(\neg x_{1} \vee x_{2}\right) \wedge\left(\neg x_{1} \vee \neg x_{2}\right)$. Matching edges are bold and all non-matching edges have weight one.

Note that, by losing the bipartition property, we can use Construction 3 to show that it is hard to approximate $\mathrm{SBC}$ on subcubic graphs to any factor better than $\left.\left(7\left(\epsilon_{4}-1\right)\right) /\left(65 \cdot \epsilon_{4}\right)\right) \approx 1.000056$ using [?]. However, we show in the next subsection how to obtain a better lower bound under the weight score for such graphs.

\subsection{Reduction from MAX 2-SAT}

We now present an $L$-reductions from the classical problem MAX 2-SAT to Semi-Brutal Cut under both scores.

MAX 2-SAT (Max 2-SAT)

Input: A boolean formula $\varphi$ in conjunctive normal form where each clause $C_{i}$ contains exactly two variables.

Question: Find an assignment maximizing the number of satisfied clauses.

Let $\varphi$ be an instance of MAX 2-SAT with $n$ variables $x_{1}, \ldots, x_{n}$ and $m$ clauses $C_{1}, \ldots, C_{m}$. For each variable $x_{i}$, let $\psi_{i}$ be the list of indices $\ell$ such that $C_{\ell}$ contains $x_{i}$. Let $\left(G^{*}, M^{*}, \omega\right)$ be a solution graph and $u$ be a vertex of $G^{*}$, we denote by $\omega(u)$ the sum of the weight of the non-matching edges incident to $u$.

Construction 4 Let $\varphi$ be an instance of MAX 2-SAT. We construct the following solution graph $\left(G^{*}, M^{*}, \omega\right)$.

1. For each $x_{i}$, construct a matching edge $u_{i} \bar{u}_{i}$ (variable edge).

2. For each clause $C_{j}$, construct a matching edge $v_{j}^{1} v_{j}^{2}$ (clause edge).

3. For each clause $C_{j}$, let $x_{k}$ be the $t^{\text {th }}$ variable of the clause. If $x_{k}$ occurs positively in the clause, then add the edge $v_{j}^{t} u_{k}$ and $\omega\left(v_{j}^{t} u_{k}\right)=1$. Otherwise, add the edge $v_{j}^{t} \bar{u}_{k}$ with $\omega\left(v_{j}^{t} \bar{u}_{k}\right)=1$.

4. Finally, for each variable matching edge $u_{i} \bar{u}_{i}$, add a matching edge $s_{1}^{i} s_{2}^{i}$. If $\omega\left(u_{i}\right)<\omega\left(\bar{u}_{i}\right)$, add an edge $s_{1}^{i} u_{1}$ with $\omega\left(s_{1}^{i} u_{1}\right)=\omega\left(\bar{u}_{i}\right)-\omega\left(u_{i}\right)$. If $\omega\left(u_{i}\right)>$ $\omega\left(\bar{u}_{i}\right)$, add an edge $s_{1}^{i} \bar{u}_{1}$ with $\omega\left(s_{1}^{i} \bar{u}_{1}\right)=\omega\left(u_{i}\right)-\omega\left(\bar{u}_{i}\right)$.

We can suppose that no variable occurs exclusively positively or exclusively negatively in the formula, thus each matching edge except the $s_{1}^{i} s_{2}^{i}$ is ambiguous. 
An example of a graph produced by Construction 4 is given in Figure 9. A normalized solution is a solution that contains exactly one cut per ambiguous edge. The following lemma shows that we can transform any solution into a normalized solution with the same weight score.

Lemma 4. Let $X$ be a solution of a solution graph $\left(G^{*}, M^{*}, \omega\right)$. There is a normalized solution $X^{\prime}$ with score $\left(X^{\prime}\right) \leq$ score $(X)$ under the weight score.

Proof. Since $X$ is a solution of SBC, after removing all non-matching edges incident to a cut, all ambiguous edges are clean. We construct $X^{\prime}$ by choosing one degree-one vertex per ambiguous edge. Clearly, $X^{\prime}$ is a solution and since each edge removed by $X^{\prime}$ is also removed by $X$, we have $\operatorname{score}\left(X^{\prime}\right) \leq \operatorname{score}(X)$.

In this, we suppose that each solution is normalized under the weight score. If a cut in a clause edge $v_{j}^{1} v_{j}^{2}$ is adjacent to a cut in a variable edge, then we say that the clause edge $v_{j}^{1} v_{j}^{2}$ is satisfied. If no extremity of a clause edge $v_{j}^{1} v_{j}^{2}$ is adjacent to a cut in a variable edge, we say that the clause edge $v_{j}^{1} v_{j}^{2}$ is unsatisfied.

Definition 2. Let $\varphi$ be a MAX 2-SAT instance, let $\left(G^{*}, M^{*}, \omega\right)$ be the graph produced by Construction 4 , and let $X$ be a normalized solution for it. An assignment $S$ for $\varphi$ corresponds to $X$ if, for all matching edges $u_{i} \bar{u}_{i}$, we have $u_{i} \in X \Rightarrow S\left(x_{i}\right)=1$ and $\bar{u}_{i} \in X \Rightarrow S\left(x_{i}\right)=0$.

Lemma 5. Let $X$ be a normalized solution for $\left(G^{*}, M^{*}, \omega\right)$, produced by Construction 4 and let $S$ be its corresponding assignment. Let $m^{\prime}$ be the number of unsatisfied clauses in $S$. There is a solution $X^{\prime}$ such that score $\left(X^{\prime}\right)=2 m+m^{\prime} \leq$ score $(X)$ and $S$ is the corresponding assignment of $X^{\prime}$.

Proof. Suppose there is a clause edge $v_{j}^{1} v_{j}^{2}$ that is neither satisfied nor unsatisfied. Thus, there is a cut vertex adjacent to $v_{j}^{1} v_{j}^{2}$ that is not adjacent to the cut vertex of $v_{j}^{1} v_{j}^{2}$. Suppose that the cut vertex in $v_{j}^{1} v_{j}^{2}$ is $v_{j}^{1}$. We can take $X^{\prime}=X \cup\left\{v_{j}^{2}\right\}-v_{j}^{1}$. Since the edge incident to $v_{j}^{2}$ is already removed, we have $\operatorname{score}\left(X^{\prime}\right) \leq \operatorname{score}(X)$. $S$ is the corresponding assignment of $X^{\prime}$ since we do not change the cuts in the variable edges. Hence, we can suppose that $X^{\prime}$ does not contain any clause edge that is neither satisfied nor unsatisfied.

Let $u_{i} \bar{u}_{i}$ be a variable edge. We have $\omega\left(u_{i}\right)=\omega\left(\bar{u}_{i}\right)=\left|\psi_{i}\right|$. Thus, the sum of the weight removed by the cuts in the variable edges is equal to $\sum_{i \leq n}\left|\psi_{i}\right|=2 m$. Let $v_{j}^{1} v_{j}^{2}$ be a clause edge. If $v_{j}^{1} v_{j}^{2}$ is satisfied, then its cut does not increase the weight of $X^{\prime}$ since the non-matching edge incident to this cut is already removed by the cut in the variable edge. If $v_{j}^{1} v_{j}^{2}$ is unsatisfied, then the cut in $\left\{v_{j}^{1}, v_{j}^{2}\right\}$ increases by one the weight of $X^{\prime}$. Since the sum of weight removed by the cuts in the unsatisfied clause-edges correspond to the number of unsatisfied clause, we have $\operatorname{score}\left(X^{\prime}\right)=2 m+m^{\prime}$.

Theorem 10. Semi-Brutal Cut cannot be approximated to any factor better than $1+\frac{\epsilon_{2}-1}{3 \cdot \epsilon_{2}}$ under the weight score.

We have $\epsilon_{2} \approx 1.06$, if the Unique Game Conjecture is true [?] and $\epsilon_{2}=22 / 21$, if $\mathcal{P} \neq \mathcal{N} \mathcal{P}[?]$. 
Proof. First, we can see that a random assignment satisfies each clause with probability $3 / 4$ and hence it is not hard to find an assignment that satisfies $3 \mathrm{~m} / 4$ clauses, yielding

$$
O P T(\varphi) \geq 3 m / 4 .
$$

Similarly to proof of Theorem 9 , we show that Construction 4 constitutes an $L$ reduction. Let $f$ be a function transforming any instance $\varphi$ of MAX 2-SAT into an instance $I$ of Semi-Brutal CUT as above. Let $X$ be a feasible normalized solution for $I$ corresponding to the property of Lemma 5 . And let $g$ be the function that construct the assignment of $\varphi$ which corresponds to $X$. Hence,

$$
O P T(I) \leq 2 m+m / 4 \stackrel{(4)}{\leq} 3 \cdot O P T(\varphi),
$$

and by Lemma 5, we have

$$
3 m=\operatorname{val}(g(X))+\operatorname{val}(X)=O P T(I)+O P T(\varphi) .
$$

Thus, we constructed an $L$-reduction with $\alpha=3$ and $\beta=1$. Since $\epsilon_{2} \cdot \operatorname{val}(g(X))<$ $\operatorname{OPT}(\varphi)$, we conclude

$$
\operatorname{val}(X) \geq\left(1+\frac{\epsilon_{2}-1}{3 \cdot \epsilon_{2}}\right) \cdot \operatorname{OPT}(I)
$$

MAX 2-SAT(3) is restricted subproblem of MAX 2-SAT where the number of occurrences of the variable is bounded by three. ?] show that MAX 2-SAT(3) cannot be approximated to a factor better than $\epsilon_{2}^{\prime} \leq 2012 / 2011$ unless $\mathcal{P}=\mathcal{N} \mathcal{P}$. In that subproblem, the maximum degree of the graph provided by Construction 4 is restricted to three. Using the same arguments as for Theorem 10, we obtain:

Theorem 11. It is $\mathcal{N} \mathcal{P}$-hard to approximate Semi-Brutal CUT within any ratio better than $\left(1+\frac{\epsilon_{2}^{\prime}-1}{3 \cdot \epsilon_{2}^{\prime}}\right)$ under the weight score, even on subcubic graphs.

Samewise, we can use the inapproximability result on MAX 2-SAT(3) to build a $L$-reduction to Semi-Brutal Cut under the cut score on subcubic graphs.

Theorem 12. It is $\mathcal{N} \mathcal{P}$-hard to approximate Semi-Brutal Cut within any ratio better than $\left(1+\frac{9\left(\epsilon_{2}^{\prime}-1\right)}{11 \cdot \epsilon_{2}^{\prime}}\right)$ under the cut score, even on subcubic graphs.

Proof. Let $\left(G^{*}, M^{*}, \omega\right)$ be a solution graph produced by Construction 4 and let $X$ be an optimal solution in $\left(G^{*}, M^{*}, \omega\right)$ for SBC under the cut score. Let $u_{i} \bar{u}_{i}$ be a variable edge, suppose by symmetry that $x_{i}$ occurs two times positively and one time negatively. Suppose that $u_{i} \bar{u}_{i}$ does not contain a cut. If the neighbor $v_{j^{\prime}}^{t^{\prime}}$ of $\bar{u}_{i}$ belongs to $X$, then we can swap $v_{j^{\prime}}^{t^{\prime}}$ and $\bar{u}_{i}$ in $X$. Otherwise, the two neighbors $v_{j}^{t}$ and $v_{j^{\prime}}^{t^{\prime}}$ belong to $X$ and then the set of cuts $X^{\prime}=X \cup\left\{u_{i}\right\} \backslash\left\{v_{j}^{t}, v_{j^{\prime}}^{t^{\prime}}\right\}$ is also a solution of SBC and $\left|X^{\prime}\right|<|X|$, contradicting the optimality of $X$. Thus, each variable edge contains at least one cut. Each cut in a variable edge can clean up to two clause edges. If a variable edge contains two cuts, then one of these 
cuts only serves to clean one clause edge $v_{j}^{1} v_{j}^{2}$, and then we can transfer this cut into $v_{j}^{1} v_{j}^{2}$. Hence, we can suppose that each variable edge contains exactly one cut. Then, the number of cuts in the clause edges corresponds to the number of unsatisfied clauses in the corresponding assignment of $X$. Note that, if a set of cuts is not optimal, it is easy to transform it into another set with a better cutscore and such that each variable edge contains exactly one cut. The number of variables in a MAX $2-\mathrm{SAT}(3)$ is equal to $2 \mathrm{~m} / 3$. Let $f$ denote the function that transforms any instance $\varphi$ of MAX 2-SAT(3) into an instance of SEMI-BRUTAL CuT as in Construction 4 . Let $X$ be a solution of SBC in $I$ that contains exactly one cut per variable edge and let $g$ be the function that construct the assignment of $\varphi$ which corresponds to $X$. We have,

$$
O P T(I) \leq 2 m / 3+m / 4 \stackrel{(4)}{\leq} 11 / 9 \cdot O P T(\varphi),
$$

and

$$
\frac{5 m}{3}=\operatorname{val}(g(X))+\operatorname{val}(X)=O P T(I)+O P T(\varphi) .
$$

We constructed an $L$-reduction with $\alpha=11 / 9$ and $\beta=1$ and we obtain:

$$
\operatorname{val}(X) \geq\left(1+\frac{9\left(\epsilon_{2}^{\prime}-1\right)}{11 \cdot \epsilon_{2}^{\prime}}\right) \cdot O P T(I)
$$

\subsection{Strict Reduction from VerTeX COVER}

Strict-reduction is the simplest type of approximation-preserving reduction [? ]. In a strict reduction, the approximation ratio $\rho_{\Pi \Pi^{\prime}}$ of a solution $y$ to an instance $f(x)$ of a problem $\Pi^{\prime}$ must be at most as good as the approximation ratio $\rho_{\Pi}$ of the corresponding solution $g(y)$ to instance $x$ of problem $\Pi$. In other words:

$$
\rho_{\Pi^{\prime}}(f(x), y) \leq \rho_{\Pi}(x, g(y)) .
$$

The proof of Theorem 8 shows that Construction 2 is a strict reduction from VERTEX COVER, which leads to the following result.

Theorem 13. If VERTEX COVER can not be approximated to a ratio better than $\rho$, then neither can SEMI-BRUTAL CUT on split graphs under the cut score.

In order to find an inapproximability result on the general case for SBC under the cut score, we reduce VERTEx COVER problem, defined in Section 6.2 and we use the following construction.

Construction 5 Let $G$ be an instance of $V C$, create a solution graph $G^{*}$ as follows: for each $v \in V(G)$, add a new path $v v^{1} v^{2} v^{3}$ and set $v v^{1}, v^{2} v^{3} \in M^{*}$. Call the resulting graph $G^{*}$ and note that $E\left(G^{*}\right) \supseteq E(G)$ and the ambiguous edges of $G^{*}$ are exactly the edges $v v^{1}$ and $\Delta\left(G^{*}\right)=\Delta(G)+1$.

Theorem 14. If VERTEX COVER can not be approximated to a ratio better than $\rho$ on graphs with bounded degree $\Delta$, then neither can SEMI-BRUTAL CUT on graph with bounded degree $\Delta+1$ under the cut score. 

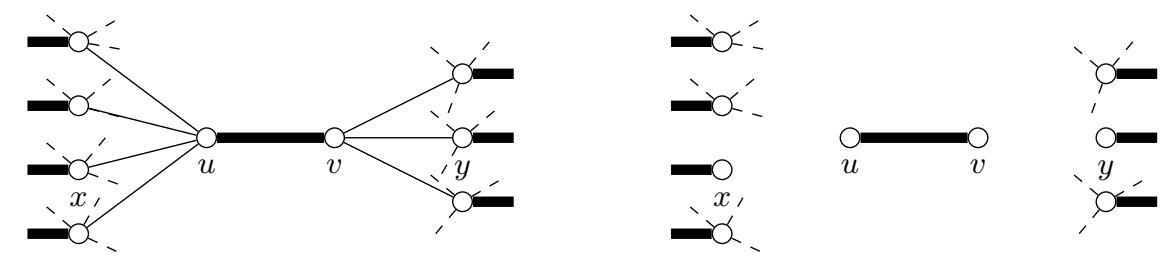

Fig. 10: A forbidden path xuvy (left) and the result of cutting all its vertices (right).

Proof. We show that $G$ has a size- $k$ vertex cover if and only if using $k$ cuts suffices to clean all matching edges in $G^{*}$.

" $\Rightarrow$ ": Let $V^{\prime}$ be a vertex-cover of $G$. Then, cutting all vertices of $V^{\prime}$ in $G^{*}$ leaves no edge of $E(G)$. The remaining graph is a collection of alternating paths of length three and, thus, all matching edges are clean.

" $\Leftarrow$ ": Let $X$ be a solution of SBC under the cut score for $G^{*}$. Let $Y:=\{v \mid$ $\left.\left\{v, v^{1}, v^{2}, v^{3}\right\} \cap X \neq \varnothing\right\}$ and note that $|Y| \leq|X|$. Now, if $Y$ is not a vertex cover of $G$, then there is an edge $u v \in E(G)$ such that $Y \cap u v=\varnothing$. Then, none of $\left\{u^{3}, u^{2}, u^{1}, u, v, v^{1}, v^{2}, v^{3}\right\}$ is cut, implying that neither $u u^{1}$ nor $v v^{1}$ is clean, contradicting the fact that $X$ is a solution of SBC.

Hence, Construction 5 is a strict reduction, transferring non-approximability results of Vertex Cover to Semi-Brutal Cut under the cut score.

VERTEX COVER is also non-approximable within a factor of 1.3606 under $\mathcal{N} \mathcal{P} \neq$ $\mathcal{P}$ [?] and within a factor $2-\epsilon, \epsilon>0$ under $\mathcal{U G C}$ [?]. Let $G$ be an instance of $\mathrm{VC}$, the maximum degree of the graph produced by Construction 5 is equal to $\Delta(G)+1$. ? ] show that if the instance of $\mathrm{VC}$ has a maximum degree three, four or five, then VC can note be approximated to a ratio better than 145/144, 79/78, $74 / 73$, respectively. Thus, this results hold for SBC under the cut score, for solution graphs with maximum degree four, five and six, respectively.

\section{Approximable Cases}

In this section, we propose one greedy approximation algorithm for each score.

Cut score: Our approximation algorithm works similarly to the well-known classical 2-approximation for VERTEX COVER that just returns the extremities of any maximal matching. Contrary to VerTex COVER, our forbidden structures are not edges, but ambiguous edges. Thus, we have to consider length-four paths containing an ambiguous edge, and we will cut all four of their vertices. In the following, we call a path xuvy forbidden if $x u$ and $v y$ are non-matching edges and $u v$ is an ambiguous edge (see Figure 10).

Lemma 6. Let $Q$ be a maximal packing of vertex-disjoint forbidden paths in $\left(G^{*}, M^{*}, \omega\right)$, let $X$ be any solution for $S B C$ under the cut score on $\left(G^{*}, M^{*}, \omega\right)$. Then, (a) cutting all vertices of $Q$ cleans all ambiguous edges in $G^{*}$ and (b) $X \cap$ $p \neq \varnothing$ for all $p \in Q$. 


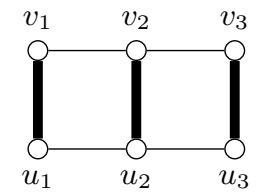

Fig. 11: Tightness of the approximation ratio for the cut-score greedy algorithm. Matching edges are bold. The approximation algorithm for the cut score provides a solution $\left\{v_{1}, v_{2}, u_{2}, u_{3}\right\}$ whereas an optimal solution is $\left\{v_{2}\right\}$.

Proof. (a): Let $H$ be the result of cutting all vertices of $Q$ in $G^{*}$. Towards a contradiction, assume that $H$ contains an ambiguous edge $u v$. By definition, there are two non-matching edges $x u$ and $v y$ in $H$. But then, the path $x u v y$ is a forbidden path, contradicting the maximality of $Q$.

(b): Let $H$ be the result of cutting all vertices of $X$ in $G^{*}$. Let $x u v y \in Q$ be a forbidden path in $\left(G^{*}, M^{*}, \omega\right)$ and assume towards a contradiction that $X \cap x u v y=\varnothing$. Then, none of the edges of xuvy are removed when cutting the vertices of $X$, that is, xuvy survives in $H$. Then, however, $u v$ is ambiguous in $H$, contradicting $X$ being a solution for $\left(G^{*}, M^{*}, \omega\right)$.

With Lemma 6, we can show that any maximal packing of forbidden paths constitutes a 4-approximation for Semi-Brutal Cut under the cut score.

Theorem 15. A 4-approximate solution to Semi-Brutal Cut under the cut score can be computed in linear time. This ratio is tight.

Proof. A packing of forbidden paths in $\left(G^{*}, M^{*}, \omega\right)$ can be computed by scanning all matching edges $u v$ and, if $u v$ is ambiguous, then $x u v y$ is a forbidden path for any non-matching edges $x u$ and $v y$. By removing $x, u, v$, and $y$ from $G^{*}$, we make sure that the resulting packing is vertex-disjoint. Thus, such a packing can be produced in linear time.

Let $Q$ be any maximal vertex-disjoint packing of forbidden paths in $\left(G^{*}, M^{*}\right.$, $\omega)$. By Lemma 6(a), the vertices of $Q$ form a solution for SBC. To show that this solution is 4-approximate, consider any optimal solution $X$ for $\left(G^{*}, M^{*}, \omega\right)$. By Lemma $6(\mathrm{~b}), X$ intersects each path in $Q$. Since the paths in $Q$ are mutually vertex disjoint and each of them contains exactly four vertices, we conclude that $Q$ contains at most four times as many vertices as $X$. The ratio is tight, as shown by Figure 11 .

Weight score: Let $\left(G^{*}, M^{*}, \omega\right)$ be a solution graph and let $X \subseteq E \backslash M^{*}$ be a set of non-matching edges. For a vertex $v$, we let $\omega_{X}(v)$ denote the sum of the weights of all non-matching edges incident with $v$ that are not in $X$. More formally, we define $\omega_{X}(v):=\sum_{e \in E \backslash\left(M^{*} \cup X\right)} \omega(e) \cdot \chi_{e}(v)$, where $\chi_{e}(v):=|e \cap\{x\}|$ is the characteristic function of $e$. The principle of our algorithm is to successively visit each ambiguous edge and cut the edges incident to the extremity with the lowest value of $w_{S}$, where $S$ contains all previously cut edges. 


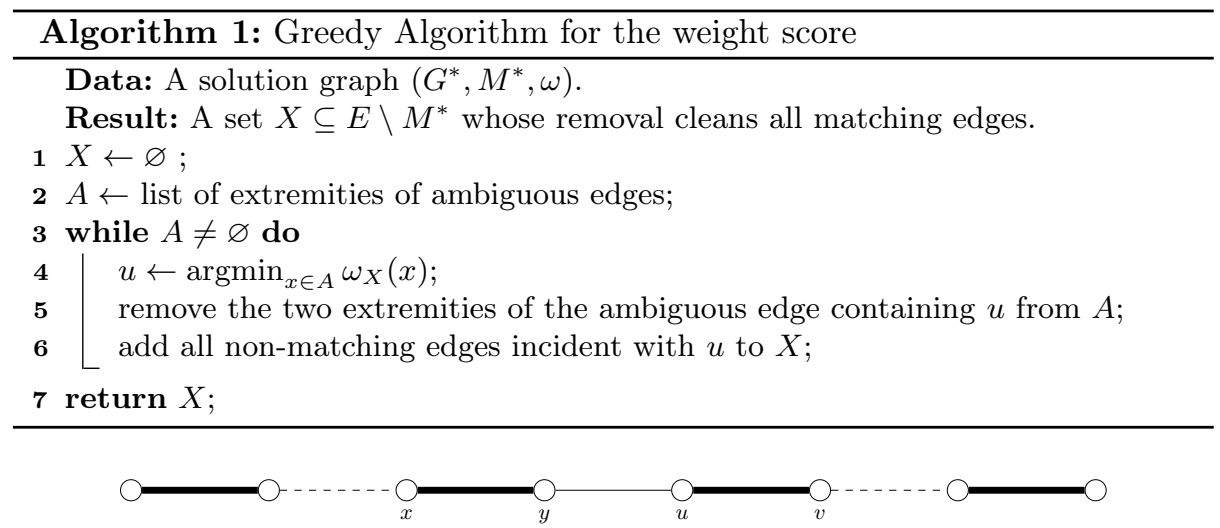

Fig. 12: Tightness of the approximation ratio for the weight-score greedy algorithm. Edges are bold $\left(\in M^{*}\right)$, solid $\left(\in X_{\text {opt }}\right)$ or dashed $(\in X)$ and all edges have weight one. Thus, $\omega(X)=2$ and $\omega\left(X_{o p t}\right)=1$.

Theorem 16. In $\mathcal{O}((|V|+|E|) \log |V|)$ time, Algorithm 1 computes a solution for SEMI-BRUTAL CUT under the weight score with an approximation ratio of 2 and this ratio is tight.

Proof. Since each time some extremities are removed from $A$, the ambiguous edge they belonged to has been cleaned, there are no more ambiguous edge remaining when $A=\varnothing$. Thus, the set $X$ that is returned is indeed a solution. Let $X_{\text {opt }}$ be an optimal solution. Let $u v$ denote the ambiguous edge of $G^{*}$ considered in step $i$ of Algorithm 1, let $X_{i}$ be the set of edges added to $X$ in step $i$. If $X_{\text {opt }}$ contains all non-matching edges incident to $u$, then let $Q_{i}$ contains them. Otherwise, $X_{\text {opt }}$ contains all non-matching edges incident to $v$, and we let $Q_{i}$ contain those. Then, $\omega\left(X_{i}\right) \leq \omega\left(Q_{i}\right)$ for all $i$ and, thus, $\omega(X) \leq \sum_{i} \omega\left(Q_{i}\right)$. Further, $\bigcup_{i} Q_{i}=X_{o p t}$ and, since each edge of $G^{*}$ occurs in at most two sets $Q_{i}$, we conclude $\sum_{i} \omega\left(Q_{i}\right) \leq 2 \omega\left(X_{\text {opt }}\right)$. The claimed approximation factor of two follows and, by Figure 12, it is tight.

Concerning the running time, the list of ambiguous edges is build in $\mathcal{O}(|E|+$ $|V|)$ with a depth-first search algorithm. The sorting of this list can be done in $\mathcal{O}(|V| \log |V|)$. Maintaining the sorting of the list at each cut yields a $\mathcal{O}((|V|+$ $|E|) \log |V|)$.

Corollary 4. Semi-Brutal Cut is $\mathcal{A} \mathcal{P} \mathcal{X}$-complete under both scores.

\section{Conclusion}

In this paper, we present complexity and approximation results obtained for a theoretical problem occurring in modern-day production of genomic sequences. We consider two variants of the problem, depending on the optimality criterion (number of cuts vs. weight of cuts). We show that the complexity of both variants 
depend heavily on the input topology. To this end, we explore the demarcation line between polynomial-time computability and $\mathcal{N} \mathcal{P}$-hard cases for sparse and dense classes of graphs. Finally, we present simple constant-factor approximation algorithms for both optimization goals. Interesting open questions include the existence and design of efficient FPT algorithms, and/or kernel techniques.

Acknowledgement: This work was partially supported by the Région Occitanie.

\section{References}

